\title{
Feedback-Assisted Correlated Packet Transmission with A Helper
}

\author{
Ade Irawan, Member, IEEE, and Tad Matsumoto, Fellow, IEEE
}

\begin{abstract}
In this paper, we analyze the impact of source correlation on the diversity and coding gains of a retransmission system where we aim to recover $M$ erroneously received packets only by transmitting one helper packet utilizing the source correlation among the packets. This system is referred to as $M$-in- 1 helper transmission. The helper packet is constructed simply by taking binary exclusive-OR of the $M$ erroneously received information packets, notified via the feedback channel. To identify the trade-off between source correlation and performance gain due to coding and diversity, we start our investigation with in-depth analyses on rate regions and outage probabilities with $M=\{2,3\}$. We also evaluate the influence of unequal power and/or redundancy allocations between the helper and information packets. Finally, we provide the analytical results on achievable diversity order with arbitrary integer values of $M$. It is shown that $M$-in- 1 helper transmission can always achieve $M$-th order diversity. Furthermore, $(M+1)$-th order diversity can be achieved with $M$ being odd when the source correlation is very high; however, it cannot be achieved with $M$ being even.
\end{abstract}

Index Terms-outage probability, admissible rate region, source correlation, feedback, HARQ, helper

\section{INTRODUCTION}

An enormous number of multimedia contents transfer impose excessive transmission problems in wireless communication networks. Such trend of the increasing data transfer demands is expected to grow continuously at an exponential order of the speed [1]. The network components in such communications systems commonly have data storage, based on which multiple streams are formed, for example, the multiple observations of the same object [2], [3], as illustrated in Fig. 1. As a consequence, the server stores correlated packets. The correlation among the information packets at the server exists, not only in the form of spatial data correlation between the information streams obtained from the different viewpoints but also the temporal data correlation between packets acquired consecutively by the same sensor [2].

Copyright (c) 2015 IEEE. Personal use of this material is permitted. However, permission to use this material for any other purposes must be obtained from the IEEE by sending a request to pubs-permissions@ieee.org.

Ade Irawan is currently with the Department of Computer Science, Universitas Pertamina, Indonesia. The research presented in this paper was undertaken when he was with Japan Advanced Institute of Science and Technology (JAIST), Japan. Email: adeirawan@universitaspertamina.ac.id.

Tad Matsumoto is with the School of Information Science, JAIST, Japan, and also with Center for Wireless Communication, University of Oulu, FI90014 Finland. Email: matumoto@jaist.ac.jp

This work has been performed in part in the framework of the FP7 project ICT-619555 RESCUE (Links-on-the-fly Technology for Robust, Efficient and Smart Communication in Unpredictable Environments) which is partly funded by the European Union.

This work has been also performed in part under JSPS Kakenhi (B) 15H04007, and Academy of Finland NETCOBRA project.

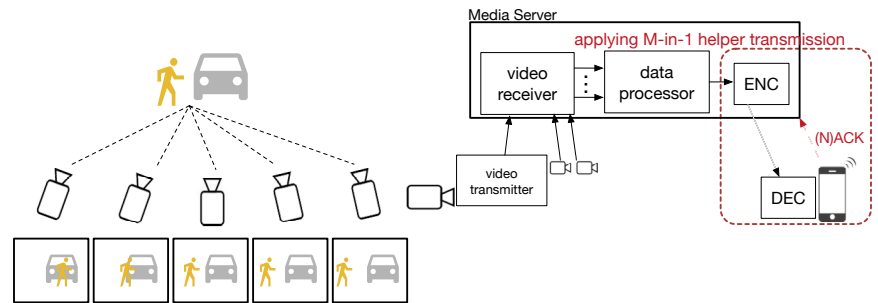

Fig. 1: Media server collects and processes the correlated packets.

In practice, Long Term Evolution (LTE) adopts H.265-High Efficiency Video Coding (HEVC) [4] to achieve high video compression. It is enabled by removing temporal data correlations within a picture and between pictures of a video sequence, using intra and inter prediction techniques, respectively [5]. The intra prediction assumes that the visual property of a picture region is similar to that in its neighborhood, while the inter prediction assumes a significant part of the pictures in a video sequence consists of objects which move in the scene. By exploiting the temporal data correlations, HEVC can provide bitrate savings of approximately $59 \%$ in average, compared to its predecessor, H.264-Advanced Video Coding (AVC), for the same observed subjective quality [6].

Theoretically, if the temporal data correlation exists, the longer the observation, the more compression possible, and it can be compressed until the entropy rate of the source. However, (1) extremely long packet size causes high decoding latency, (2) the fixed packet size fits the block fading channel assumption [7], and (3) in many practical cases, telecommunication technology standard commonly fixes the length of the packet. This indicates that the information in each packet is correlated if we set the packet size to meet the block fading assumption [7]. Therefore, instead of compressing the information to the close-entropy-rate at the transmitter, our aim is to exploit the correlation among the packets to reduce the required signal-to-noise power ratio (SNR) at the receiver. ${ }^{1}$

With the enormous demands, designing very highly reliable data transfer mechanism is of crucial importance in many cases [8]. Hybrid automatic repeat request (HARQ) [9]-[11] is commonly used for the systems requiring high-reliability. With HARQ, the receiver sends back to the transmitter an acknowledgement (ACK) or a negative ACK (NACK), depending on whether or not the received packet contains no errors after

\footnotetext{
${ }^{1}$ Note that we assume capacity-achieving channel coding in this paper, even though it requires large enough length, such as turbo codes or low-density parity-check (LDPC) codes. This is only for the ease of analysis.
} 
decoding, respectively. The transmitter then decides whether to transmit new packets or to retransmit the packets found to be received in error, respectively.

With HARQ, incremental redundancy technique is known as an effective scheme to achieve high throughput [12]. The aim of this scheme is to inherently make an adaptive adjustment between the unknown received SNR and Forward Error Correction (FEC) code rate through accumulative redundancy transmissions [13]. However, it still requires per-packet ACK/NACK feedback, which causes a detrimental effect when packet-wise transmitter-receiver synchronization is somehow disrupted. Various block-wise feedback based techniques using rateless coding have been proposed to eliminate this drawback [14]-[16].

Instead of block-wise feedback, however, the per-packet feedback based techniques have been revisited recently, where its effectiveness has been investigated by utilizing the network coding techniques [17]-[19]. The broadcast transmission in [17] and the multiple unicast schemes in [18] use the binary exclusive-OR (XOR) network coding to reduce the number of retransmissions compared with conventional HARQ schemes. Authors in [19] apply random network coding for point-to-point communication to further reduce unnecessary redundancy transmission. However, none of those techniques described in [17]-[19] take into account the impact of the source correlation.

In the per-packet feedback system, utilizing capacityachieving code for the transmission over block fading channel [7] ensures that the packets can be received with arbitrarily low error probability if the instantaneous received SNR is larger than the threshold SNR supported by the code. However, the received instantaneous SNR varies in fading channels, and hence the fading variation dominates the average performance, such as the decay of the outage probability curve represented by the diversity order.

The observation described above invokes fundamental interest that how HARQ can well utilize the source correlation knowledge and how the redundancy should be constructed. This interest motivates us to investigate the relationship between outage probability, achievable diversity order, and source information correlation. To the best of our knowledge, how to utilize the source correlation knowledge at the receiver to improve the performance of the HARQ systems has not yet well been investigated.

\section{A. M-in-1 Helper Transmission}

In this paper, we consider a per-packet feedback system for correlated information sources which are transmitted over independent block Rayleigh fading channels. Also, we limit the redundancy packet transmissions to be one for $M$ correlated information sources. Each packet is encoded by a capacity-achieving code at a certain specified instantaneous SNR. With this assumption, any packets received in error if the channel capacity ${ }^{2}$ falls below the entropy of each packet. The receiver notifies the decoding failure to the transmitter via the feedback channel. Then, the transmitter sends a helper packet, which is formed by utilizing the XOR operation to the $M$ unrecovered information packets at the receiver. ${ }^{3}$ Therefore, the system considered in this paper is regarded as two-dimensional channel coded packet-wise transmission, horizontal and vertical codes. The horizontal code is the packet-wise capacity-achieving code, and the vertical code is binary single parity check code over $M$ information packets. This system is referred to as $M$-in- 1 helper transmission in this paper.

\section{B. Contributions of This Work}

We start our investigation with in-depth analyses on the rate regions in a static additive white Gaussian noise (AWGN) channel with $M=\{2,3\}$ to simplify the analyses on the difference in the rate regions between the feedback and nonfeedback systems. We also analyze the impact of the source information correlation to the achievable diversity order of the system, with $M$ being odd and even.

The analyses are based on the theorem of source coding with side information [20], [21], where the authors consider the problem of two sources performing independent encoding and joint decoding; the decoder wishes to reconstruct one source asymptotically lossless so that the other serves as a helper. However, it can be generalized to an arbitrary number of sources as presented, for example, by Theorem 10.4 in [22].

Given the derived inadmissible rate region in this paper, we then derive the upper bound of the outage probability of the system in block Rayleigh fading channels. ${ }^{4}$ Finally, we generalize the analyses of achievable diversity order to any integer $M$.

A technique very close to the idea investigated in this paper is the orthogonal multiple access relay channel in [23], referred to as o-MARC for notation convenience. The authors of [23] aim to utilize the same theorem in [22] for analyzing the oMARC cooperative wireless communications. They investigate the admissible rate region of the o-MARC system in static AWGN channels and uses the rate region for deriving the outage probabilities in independent block fading channels.

A fundamental difference in the rate region analysis between the technique proposed in this paper and o-MARC is that with the proposed technique, that utilizes the feedback information, the rate region supported by the channel larger than the entropy does not have to be taken into account, while without feedback it has to be. This is because the packet is always received correctly in this case due to the use of a capacity-achieving code. Hence, such packets do not have to be included when

\footnotetext{
${ }^{2}$ More specifically, it is the channel capacity corresponding to the specified SNR, divided by the signaling spectrum efficiency which is including channel coding rate and modulation multiplicity, according to Shannon's sourcechannel separation theorem. Unless otherwise stated, however we use the terminology "capacity" for the simplicity.

${ }^{3}$ Afterward, we use terminology NACK-ed packet to refer the packet that are unable to be recovered at the receiver by independent (packet-by-packet) decoding.

${ }^{4}$ The outage probability of the systems with $M>3$ may be possible to be derived if we can solve the difficulty of managing $M$ dimensions rate region.
} 
forming the helper packet. This fact makes a fundamental difference in the achievable diversity order of the outage curve between the proposed technique and o-MARC; with the proposed technique, at least $M$-th order diversity can be achieved, as shown in Section VI, while o-MARC, the achievable diversity order is always two.

We summarize the contributions of this paper as follows:

- Presenting theoretical derivation of inadmissible rate region and upper bound of outage probability of the $M$-in- 1 helper transmission system by considering the case that the per-packet entropy is larger than the channel capacity at a certain specified instantaneous SNR.

- Analyzing the effects of the source information correlation and the bit error rate of the helper packet on the inadmissible rate region and the upper bound of the outage probability of the $M$-in-1 helper transmission system.

- Providing proof for $M$-in-1 helper transmission the achievabilities of $M$ th order diversity with arbitrary $M$. Furthermore, $(M+1)$-th order diversity can be achieved with $M$ being odd when the source correlation is very high.

\section{Notation}

The following notations are adopted in this paper. Vectors are expressed with bold lowercase and scalars with standard text notation. We use $b \in \boldsymbol{b}$ where $b$ is a bit in the binary vector $\boldsymbol{b}$. Probability is expressed by $\mathbb{P}(\cdot)$. The $\oplus$ indicates binary XOR, and $*$ denotes convolution operation, e.g., $\alpha * \beta=$ $\alpha(1-\beta)+(1-\alpha) \beta . H_{b}(\cdot)$ denotes the binary entropy function, where $H_{b}(\alpha)=-\alpha \log _{2} \alpha-(1-\alpha) \log _{2}(1-\alpha)$. Afterward, we use $M 2$ and $M 3$ to denote the schemes with $M=2$ and the $M=3$, respectively.

\section{Paper Organization}

The rest of this paper is organized as follows. Section II introduces the system model of $M$-in- 1 helper transmission assumed in this paper. The information theoretical limit of the system is given in Section III. Furthermore, the inadmissible rate region with $M=2$ and $M=3$ are provided in Section III-A and Section III-B, respectively. Then, the outage probabilities and upper bound approximation are theoretically derived in Section IV: Section IV-A and Section IV-B show the derivation of the outage probabilities with $M=2$ and $M=3$, respectively, which are further detailed in Appendix A and Appendix B. With the results obtained from Section IV, Section V presents results of the numerical analyses, and demonstrates the influence of unequal power and/or redundancy allocations between the helper and information packets. Afterward, we generalize the achievable diversity order analyses for any integer $M$ in Section VI. Finally, concluding remarks are given in Section VII.

\section{System ModeL}

We consider a point-to-point wireless communication system where the transmitter is a binary source generating $\mathcal{N}$
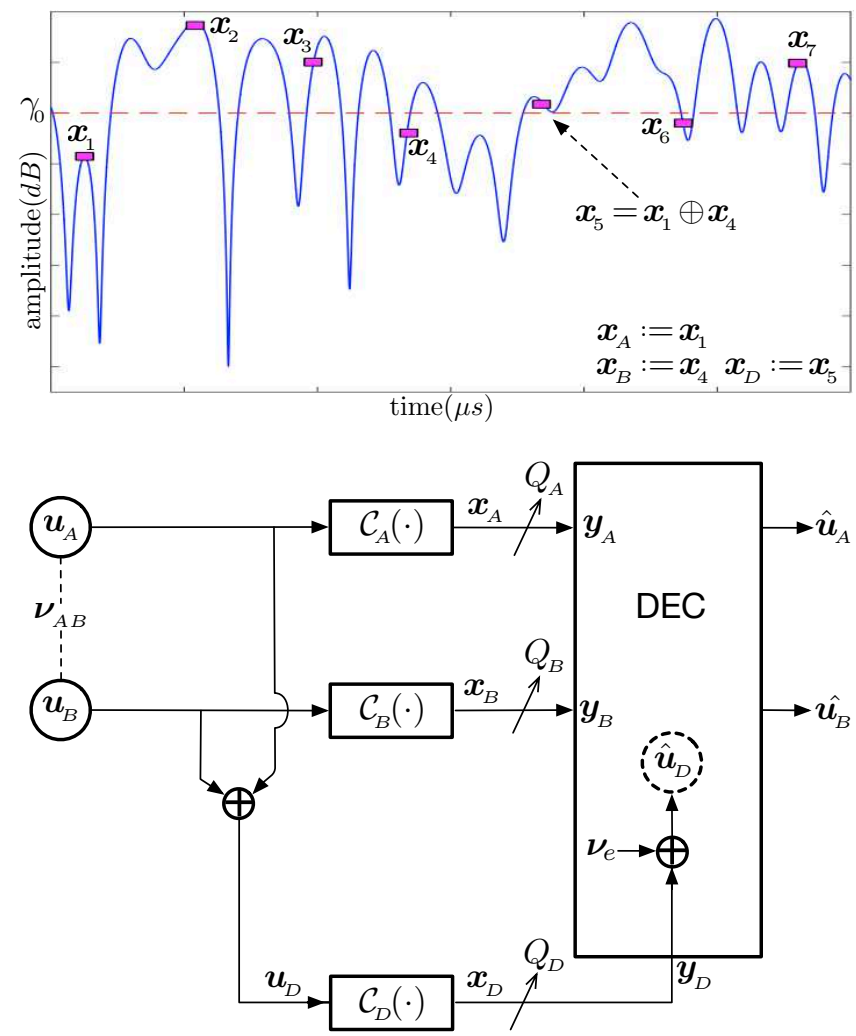

(a) System model of $M 2$.
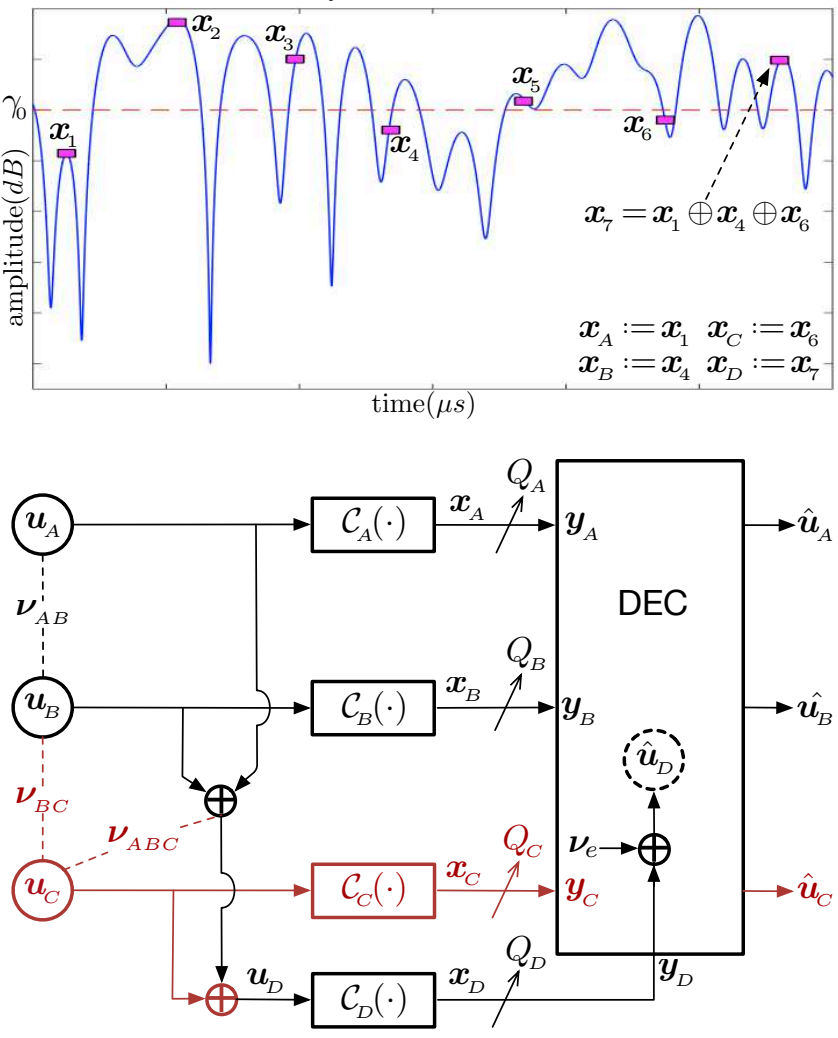

(b) System model of $M 3$.

Fig. 2: Transmission over fading channel and the corresponding system model with $M 2$ and $M 3$. Note that the feedback signal is not shown in the figure for the sake of simplicity. 
information packet $\mathbf{u}$, i.e., $\mathbf{u}=\left\{\mathbf{u}_{1}, \mathbf{u}_{2}, \cdots, \mathbf{u}_{\mathcal{N}}\right\}$. Each information packet is a binary sequence with rate $R_{n}$ and $\mathbb{P}\left(\mathbf{u}_{n}[k]=0\right)=\mathbb{P}\left(\mathbf{u}_{n}[k]=1\right)=0.5$, where $n=$ $\{1,2, \cdots, \mathcal{N}\}, k=\{1,2, \cdots, \mathcal{K}\}$, and $\mathbf{u}_{n}[k]$ denotes the $k$ th bit in $\mathbf{u}_{n}$ having the length of $\mathcal{K}$ bits. A function $\mathcal{C}_{n}(\cdot)$ encodes and modulates $\mathbf{u}_{n}$ in such a way that the spectrum efficiency of the signaling scheme, including channel coding rate and modulation multiplicity, is $Q_{n}$. The encoded packet $\boldsymbol{x}_{n}=\mathcal{C}_{n}\left(\mathbf{u}_{n}\right)$ is then transmitted via a channel assumed to be suffering from block Rayleigh fading.

The received packets can be expressed as $\boldsymbol{y}_{n}=h_{n} \cdot \boldsymbol{x}_{n}+\boldsymbol{v}_{n}$, where $h_{n}$ and $\boldsymbol{v}_{n}$ represent the complex channel gain and the zero mean AWGN vector with variance $\sigma_{n}^{2}$, respectively. With the block Rayleigh fading assumption, $h_{n}$ is constant within a block, and varies independently block-by-block; it has Rayleigh-distributed amplitude $\left|h_{n}\right|$ with $E\left[\left|h_{n}\right|^{2}\right]=1$. The instantaneous received SNR for the transmission of the block $\boldsymbol{x}_{n}$ is then given by $\gamma_{n}=\left|h_{n}\right|^{2} \cdot \Gamma_{n}$, where $\Gamma_{n}$ is the average SNR. The probability density function $(p d f)$ of $\gamma_{n}$ is

$$
p\left(\gamma_{n}\right)=\frac{1}{\Gamma_{n}} \exp \left(-\frac{\gamma_{n}}{\Gamma_{n}}\right) .
$$

The system utilizes a simple stop-and-wait HARQ protocol where the receiver sends an ACK to indicate successful packet decoding, otherwise, NACK to indicate the retransmission request. The transmitter is assumed to have a buffer with size $M$ to store $M$ NACK-ed packets.

With $M 2$, let the $M$ NACK-ed packets be $\boldsymbol{u}_{A}$ and $\boldsymbol{u}_{B}$, where $1 \leq A<B \leq \mathcal{N}$, and the corresponding rates and signaling spectrum efficiencies are $R_{A}$ and $Q_{A}$, and $R_{B}$ and $Q_{B}$, respectively. After receiving NACK twice, i.e., the buffer becomes full, the transmitter transmits a helper packet, represented by $\boldsymbol{u}_{D}=\boldsymbol{u}_{A} \oplus \boldsymbol{u}_{B}$, by utilizing rate $R_{D}$ code with signaling spectrum efficiency $Q_{D}$, as shown in Fig. 2a.

Likewise, with $M 3$, let the $M$ NACK-ed packets be denoted as $\boldsymbol{u}_{A}, \boldsymbol{u}_{B}$, and $\boldsymbol{u}_{C}$, where $1 \leq A<B<C \leq \mathcal{N}$, the corresponding rate and signaling spectrum efficiency of $\boldsymbol{u}_{C}$ is $R_{C}$ and $Q_{C}$, respectively. An additional XOR operation with the packet $\boldsymbol{u}_{C}$ is included in the helper packet so that $\boldsymbol{u}_{D}=$ $\boldsymbol{u}_{A} \oplus \boldsymbol{u}_{B} \oplus \boldsymbol{u}_{C}$, as shown in Fig. 2b. After receiving the helper packet, the receiver again decodes all NACK-ed packets jointly with the estimated helper packet $\hat{\boldsymbol{u}}_{D}$. Since the receiver does not aim to successfully decode $\boldsymbol{u}_{D}, \hat{\boldsymbol{u}}_{D}$ may contain some errors. We express the correlation between $\boldsymbol{u}_{D}$ and $\hat{\boldsymbol{u}}_{D}$ by

$$
\nu_{e}=\boldsymbol{u}_{D} \oplus \hat{\boldsymbol{u}}_{D}
$$

where $\nu_{e}$ is the error vector with its bit error probability

$$
p_{e}=\mathbb{P}\left(\nu_{e}=1\right), 0 \leq p_{e} \leq 0.5 \text {. }
$$

The receiver exploits the information source correlation knowledge among the NACK-ed packets to enhance the error correction capability. The correlation is assumed to be described by the bit-flipping model [24] as:

$$
\begin{aligned}
\boldsymbol{\nu}_{A B} & =\boldsymbol{u}_{A} \oplus \boldsymbol{u}_{B}, \\
\boldsymbol{\nu}_{B C} & =\boldsymbol{u}_{B} \oplus \boldsymbol{u}_{C}, \\
\boldsymbol{\nu}_{A B C} & =\boldsymbol{u}_{A} \oplus \boldsymbol{u}_{B} \oplus \boldsymbol{u}_{C},
\end{aligned}
$$

with bit-flipping probabilities

$$
\begin{aligned}
p_{A B} & =\mathbb{P}\left(\nu_{A B}=1\right), \\
p_{B C} & =\mathbb{P}\left(\nu_{B C}=1\right), \text { and } \\
p_{A B C} & =\mathbb{P}\left(\nu_{A B C}=1\right),
\end{aligned}
$$

where $0 \leq p_{z} \leq 0.5, z \in\{A B, B C, A B C\}$. With the model given above, the value of $p_{A B C} \approx 0.5$. Let $\rho_{z}$ denotes the exact correlation, the relationship between $\rho_{z}$ and $p_{z}$ is given by

$$
\rho_{z}=1-2 p_{z} .
$$

Notice that in the extreme cases, $\rho_{z}=0$ and $\rho_{z}=1$ indicate no correlation and full correlation, respectively.

We assume the receiver knows the correlation among the information parts of the NACK-ed packets when the buffer becomes full, by utilizing, for example, a packet index with an initial correlation parameter setting with the help of higher layer protocols. However, how to estimate and/or share the correlation information is out of the scope of this paper. We also assume that correlation between the NACK-ed packets follows the Markov process, and hence the Markov chain $\boldsymbol{u}_{A} \rightarrow \boldsymbol{u}_{B} \rightarrow \boldsymbol{u}_{C}$ holds.

\section{InADMissible RATE REgion IN StATIC AWGN CHANNEL}

In this section, we identify the inadmissible rate regions of $M 2$ and $M 3$, which are defined by the set of all the possible rate vectors $\left(R_{A}, R_{B}\right)$ and $\left(R_{A}, R_{B}, R_{C}\right)$, respectively, with which the values of each rate and sum-rates do not follow the given constraints. The admissible rate region is the complement of the inadmissible rate region. First of all, we recall for both $M 2$ and $M 3$ the theoretical rate regions of which the constraints are derived from the theorem of multiple sources coding with a helper without feedback. Finally, we derive the inadmissible rate regions of the $M 2$ and $M 3$ cases with feedback, based on the protocol considered in this paper. Accordingly, an additional constraint is taken into account.

\section{A. Inadmissible Rate Region of $M 2$}

Let us first assume there is no feedback channel in the systems, as in o-MARC. In this case, the helper is transmitted following the two distinct packet transmissions regardless of whether or not the decoding is successful. The theoretical rate region is given by equations (38a)-(38d) in Appendix A [23]. Let us then assume that the constraint for the helper packet's information rate, $R_{D} \geq \theta_{2}$, is always satisfied. ${ }^{5}$ It is easily found that for an arbitrary value of $R_{D} \geq \theta_{2}$, the entire rate region for the rate-pair $R_{A}$ and $R_{B}$ can be divided into five parts, i.e. $\mathcal{A}_{w}, w=\{1,2, \cdots, 5\}$, as shown in Fig. 3 . Accordingly, the admissible region for the non-feedback case corresponds to the regions $\mathcal{A}_{2} \cup \mathcal{A}_{5}$.

Now, let us assume that the feedback channel is available. Based on the system model described in Section II, the ACK/NACK is fed back from the receiver via the feedback channel after decoding the received packets, at the receiver,

\footnotetext{
${ }^{5}$ This assumption is eliminated when deriving the outage probability in the next section, such that the variation of the rate $R_{D}$ can be taken into account.
} 


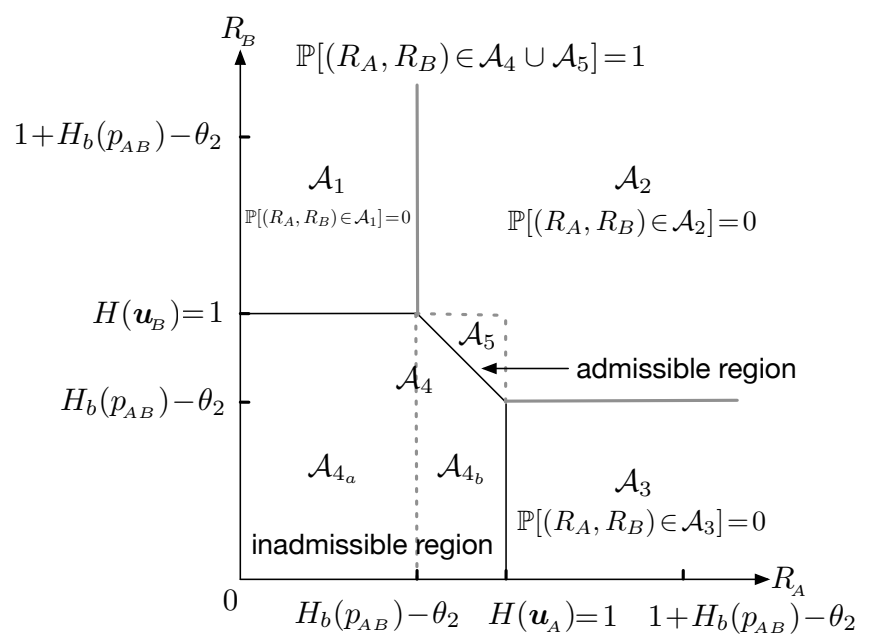

Fig. 3: Rate region of the rate pair $\left(R_{A}, R_{B}\right)$ given $R_{D} \geq \theta_{2}$ for $M 2$.

packet-by-packet. Since we assume a capacity-achieving channel code, ACK feedback indicates $R_{i} \geq H\left(\boldsymbol{u}_{i}\right), i \in\{A, B\}$, whereas NACK feedback indicates $R_{i}<H\left(\boldsymbol{u}_{i}\right)$. Therefore, when the receiver starts joint decoding after receiving the helper packet, the possible rate region is limited to $\mathcal{A}_{4}$ and $\mathcal{A}_{5}$, i.e. $\mathbb{P}\left[\left(R_{A}, R_{B}\right) \in \mathcal{A}_{1}\right]=\mathbb{P}\left[\left(R_{A}, R_{B}\right) \in \mathcal{A}_{2}\right]=\mathbb{P}\left[\left(R_{A}, R_{B}\right) \in\right.$ $\left.\mathcal{A}_{3}\right]=0$. In this case, all NACK-ed packets can be recovered only when the rate-pair, $\left(R_{A}, R_{B}\right)$, falls in the region $\mathcal{A}_{5}$. Conversely, they cannot be recovered when the rate-pair falls in the region $\mathcal{A}_{4}$, defines the inadmissible rate region with $M 2$. We divide the inadmissible rate region into two parts, $\mathcal{A}_{4_{a}}$ and $\mathcal{A}_{4_{b}}$, and hence the region can be expressed as

$$
\mathcal{A}_{4}=\mathcal{A}_{4_{a}} \cup \mathcal{A}_{4_{b}},
$$

where

$$
\begin{aligned}
\mathcal{A}_{4_{a}}= & \left\{\left(R_{A}, R_{B}\right) \mid 0 \leq R_{A}<H_{b}\left(p_{A B}\right)-\theta_{2}, 0 \leq R_{B}<1\right\}, \\
\mathcal{A}_{4_{b}}= & \left\{\left(R_{A}, R_{B}\right) \in \mathbb{R}^{+} \mid H_{b}\left(p_{A B}\right)-\theta_{2} \leq R_{A}<1,\right. \\
& \left.\quad 0 \leq R_{B}<1+H_{b}\left(p_{A B}\right)-\theta_{2}-R_{A}\right\}, \\
\theta_{2}= & H_{b}\left(p_{A B} * p_{e}\right)-H_{b}\left(p_{e}\right) .
\end{aligned}
$$

This is the most significant difference in the rate region between with and without feedback, corresponding to this paper and o-MARC, respectively.

\section{B. Inadmissible Rate Region of M3}

We use the same logic of deriving the inadmissible rate region as in the previous subsection, to derive that with the $M 3$ scheme. The admissible rate region without feedback is given by equations (40a)-(40h) in Appendix B [22]. From the equations, we get the constraints of the helper packet's information rate, $R_{D} \geq \theta_{3}$, and the sum-rate, $R_{A}+R_{B}+R_{C} \geq$ $1+H_{b}\left(p_{A B}\right)+H_{b}\left(p_{B C}\right)-\theta_{3}$.

We analyze an HARQ system with $M$-in-1 helper transmission utilizing joint decoding of a helper and three unsuccessful independently decoded packets, for which NACKs have been received via the feedback channel. It is found that for

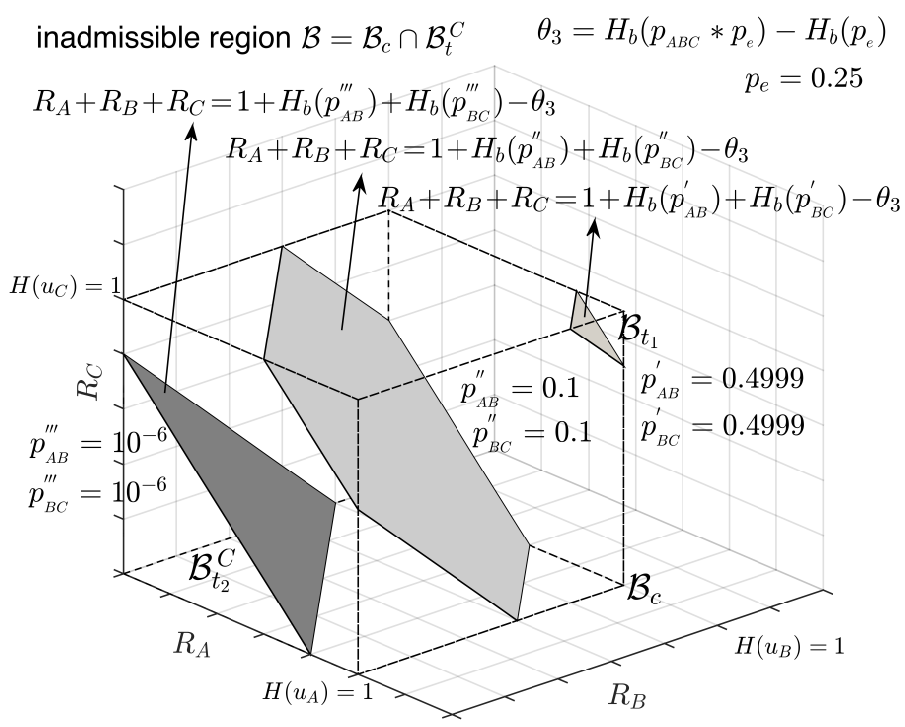

Fig. 4: Rate region of the rate vector $\left(R_{A}, R_{B}, R_{C}\right)$ given $R_{\mathrm{D}} \geq \theta_{3}$ for $M 3$.

arbitrary value of $R_{D} \geq \theta_{3}$, the inadmissible rate region of $\left(R_{A}, R_{B}, R_{C}\right)$, referred to as $\mathcal{B}$, is obtained by

$$
\mathcal{B}=\mathcal{B}_{c} \cap \mathcal{B}_{t}^{C},
$$

where $\bullet^{C}$ denotes the complement of the rate vector set in region $\bullet$, as shown in Fig. 4. The cube $\mathcal{B}_{c}$ is determined by the constraints of each single transmission, $R_{i} \geq H\left(\boldsymbol{u}_{i}\right), i \in$ $\{A, B, C\}$, whereas the $\mathcal{B}_{t}$ region is determined by the constraint of the sum-rate. $\mathcal{B}_{t}$ region being above the plain $R_{A}+R_{B}+R_{C}=1+H_{b}\left(p_{A B}\right)+H_{b}\left(p_{B C}\right)-\theta_{3}$ up to the corner $\left(H\left(\boldsymbol{u}_{A}\right)=1, H\left(\boldsymbol{u}_{B}\right)=1, H\left(\boldsymbol{u}_{C}\right)=1\right)$ of $\mathcal{B}_{c}$.

Fig. 4 shows that $\mathcal{B}_{t}$ has different geometric shape even with the same value of $p_{e}$, depending on the value of $p_{A B}$ and $p_{B C}$. For the extreme cases, we found $\mathcal{B}_{t}$ has triangular base plain, i.e. $\mathcal{B}_{t_{1}}$ corresponding to $p_{A B}=p_{B C}=0.4999$ and $\mathcal{B}_{t_{2}}$ to $p_{A B}=p_{B C}=10^{-6}$, for arbitrary value of $p_{e}$. All the aforementioned regions are given as follows.

$$
\left.\mathcal{B}_{c}=\left\{\left(R_{A}, R_{B}, R_{C}\right) \mid 0 \leq R_{A}<1,0 \leq R_{B}<1,0 \leq R_{C}<1\right\}\right\},
$$

$$
\begin{aligned}
\mathcal{B}_{t_{1}}= & \left\{\left(R_{A}, R_{B}, R_{C}\right) \in \mathbb{R}^{+} \mid 1+H_{b}\left(p_{A B}\right)+H_{b}\left(p_{B C}\right)-\theta_{3}-R_{B}\right. \\
& -R_{C} \leq R_{A}<1, H_{b}\left(p_{A B}\right)+H_{b}\left(p_{B C}\right)-\theta_{3}-R_{C} \leq R_{B}<1, \\
& \left.H_{b}\left(p_{A B}\right)+H_{b}\left(p_{B C}\right)-\theta_{3}-1 \leq R_{C}<1\right\}, \\
\mathcal{B}_{t_{2}}^{C}= & \left\{\left(R_{A}, R_{B}, R_{C}\right) \mid 0 \leq R_{A}<1+H_{b}\left(p_{A B}\right)+H_{b}\left(p_{B C}\right)-\theta_{3}\right. \\
& -R_{B}-R_{C}, 0 \leq R_{B}<1+H_{b}\left(p_{A B}\right)+H_{b}\left(p_{B C}\right)-\theta_{3}-R_{C}, \\
& \left.0 \leq R_{C}<1+H_{b}\left(p_{A B}\right)+H_{b}\left(p_{B C}\right)-\theta_{3}\right\},
\end{aligned}
$$

where $\theta_{3}=H_{b}\left(p_{A B C} * p_{e}\right)-H_{b}\left(p_{e}\right)$. Notice that with $\mathcal{B}_{t_{2}}^{C}$, the inadmissible region $\mathcal{B}=\mathcal{B}_{c} \cap \mathcal{B}_{t_{2}}^{C}=\mathcal{B}_{t_{2}}^{C}$.

\section{Outage Probability in Block Rayleigh Fading CHANNEL}

The scenario described in Introduction may arise in HARQ systems where the transmitter stores the NACK-ed packets in a buffer with a size of $M$, and a helper is transmitted 
whenever the buffer is full. For the analysis, we only focus on the buffer-full state and derive the outage probabilities in block Rayleigh fading channel based on the results of the inadmissible rate regions analyses shown in Section III. In fact, the process of how the buffer full state is reached has to be taken into account for the exact calculation of the system outage, if the information packet transmission time is fixed. In this paper, however, we ignore the process and make use of the statistically independent occurrence of the two events, decoding success and failure at the receiver. We define the outage event such that decoding of the $M$ NACK-ed packets after transmitting the helper packet is failed for the first time, and thereby the outage probability derived in this paper is an upper bound.

With the capacity-achieving channel codes assumption, the relationship between $R_{n}$ and its corresponding instantaneous SNR, $\gamma_{n}$, is given by function $\Phi_{n}\left(\gamma_{n}\right)$ as [23]

$$
R_{n}=\Phi_{n}\left(\gamma_{n}\right)=\frac{1}{Q_{n}} \log _{2}\left(1+\gamma_{n}\right),
$$

with its reverse function

$$
\gamma_{n}=\Phi_{n}^{-1}\left(R_{n}\right)=2^{R_{n} \cdot Q_{n}}-1 .
$$

Since all the transmissions are suffering from statistically independent block Rayleigh fading, the joint pdf of the instantaneous SNRs can be expressed as $p\left(\gamma_{A}, \cdots, \gamma_{D}\right)=$ $p\left(\gamma_{A}\right) \cdots p\left(\gamma_{D}\right)$.

There are three events possible in the system: (1) event of successfully decoding a packet with independent decoding, (2) event of successfully decoding $M$ packets with the joint decoding, and (3) event of unsuccessfully decoding $M$ packets with the joint decoding. Assuming the information source generates an infinite number of packets, the occurrence of those events is identical and independently distributed (i.i.d.). Therefore, the outage probability of $M$-in-1 helper transmission, $P_{\text {out }}(M)$, can be obtained by analyzing the probability that the $M$ packets in the buffer cannot be recovered with the joint decoding for the first time, which is given by (16). With independent decoding, the outage probability in block Rayleigh fading channel corresponds to the probability that the rate $R_{n}<H\left(\mathbf{u}_{n}\right)=1$ is

$$
\mathscr{P}_{n}=\int_{\Phi_{n}^{-1}(0)}^{\Phi_{n}^{-1}} \frac{1}{\Gamma_{n}} \exp \left(-\frac{\gamma_{n}}{\Gamma_{n}}\right) d_{\gamma_{n}}=1-\exp \left(-\frac{2^{Q_{n}}-1}{\Gamma_{n}}\right) .
$$

Hence, $P_{\text {out }}(M)$ can be obtained by

$$
P_{\text {out }}(M)=\left(1-\mathscr{P}_{n}\right)^{\omega}(1-\mathcal{E}(M))^{\lambda-1} \mathcal{E}(M),
$$

where $\omega$ denotes the number of the packets successfully recovered with independent decoding and $\lambda$ denotes the number of transmitted helper packet; those numbers are calculated before the receiver reaches the first unsuccessful recovery of $M$ information packets with joint decoding. $\mathcal{E}(M)$ denotes the probability that $M$ packets are unrecovered with independent decoding and the corresponding rates are falling into the inadmissible rate region. ${ }^{6}$

${ }^{6}$ Rate vectors $\left(R_{A}, R_{B}\right)$ and $\left(R_{A}, R_{B}, R_{C}\right)$ fall into regions $\mathcal{A}_{4}$ for $M 2$ and $\mathcal{B}$ for $M 3$, respectively.
Approximation (Upper Bound). The outage probability of the system is upper bounded by $(1-\mathcal{E}(M))^{\lambda-1} \mathcal{E}(M)$ because of $\left(1-\mathscr{P}_{n}\right)^{\omega} \leq 1$. However, since $(1-\mathcal{E}(M)) \leq 1$, $P_{\text {out }}(M) \leq \mathcal{E}(M)$.

Let $\mathcal{P}_{M}$ be the probability that $M$ packets are unrecovered with independent decoding, and $\mathcal{P}_{E \mid M}$ be the probability that the rate vector of the information packets falls into inadmissible region, given the fact that those packets are unrecovered with independent decoding. Then $\mathcal{E}(M)$ is given by

$$
\mathcal{E}(M)=\mathcal{P}_{E \mid M} \cdot \mathcal{P}_{M}
$$

By taking into account the impact of the channel variation in the helper transmission phase, $\mathcal{P}_{E \mid M}$ is obtained by taking average over $p_{e}$ given by

$p_{e}= \begin{cases}H_{b}^{-1}\left(H_{b}(g)-\Phi_{D}\left(\gamma_{D}\right)\right), & \text { for } \Phi_{D}^{-1}(0) \leq \gamma_{D}<\Phi_{D}^{-1}\left(H_{b}(g)\right), \\ 0, & \text { for } \gamma_{D} \geq \Phi_{D}^{-1}\left(H_{b}(g)\right),\end{cases}$

where $g=p_{A B}$ for $M 2$ and $g=p_{A B C}$ for $M 3$.

\section{A. Outage Probability with M2}

The outage probability of $M 2$ is upper bounded by $P_{\text {out }}(2) \leq \mathcal{P}_{E \mid 2} \cdot \mathcal{P}_{2}$, where

$$
\mathcal{P}_{2}=\mathscr{P}_{A} \cdot \mathscr{P}_{B}
$$

and $\mathcal{P}_{E \mid 2}$ is given by (20). The normalization factor $\mathbb{P}\left(\mathcal{A}_{4} \cup\right.$ $\left.\mathcal{A}_{5}\right)$ is defined by

$$
\begin{aligned}
\mathbb{P}\left(\mathcal{A}_{4} \cup \mathcal{A}_{5}\right) & =\int_{\Phi_{A}^{-1}(0)}^{\Phi_{A}^{-1}(1)} \int_{\Phi_{B}^{-1}(0)}^{\Phi_{B}^{-1}(1)} p\left(\gamma_{A}\right) p\left(\gamma_{B}\right) d_{\gamma_{A}} d_{\gamma_{B}} \\
& =\left[1-\exp \left(-\frac{\Phi_{A}^{-1}(1)}{\Gamma_{A}}\right)\right]\left[1-\exp \left(-\frac{\Phi_{B}^{-1}(1)}{\Gamma_{B}}\right)\right] .
\end{aligned}
$$

The probabilities in (20) are given by equations (41)-(44) in Appendix C.

\section{B. Outage Probability with M3}

As described in Section III-B, $\mathcal{B}_{t}$ has various geometric shape depending on the value of $p_{e}, p_{A B}$ and $p_{B C}$. For the sake of simplicity, we theoretically derive the outage probability of $M 3$ in the case $\mathcal{B}_{t}$ has a triangular plain, e.g. $\mathcal{B}_{t_{1}}$ and $\mathcal{B}_{t_{2}}$. The result is verified by the Monte Carlo simulation shown in Section V. Eventually, in other cases, the outage probability can be calculated by the Monte Carlo simulations.

The upper bound of the outage probability of $M 3$ is given by $P_{\text {out }}(3) \leq \mathcal{P}_{E \mid 3}\left(\mathcal{B}_{t}\right) \cdot \mathcal{P}_{3}$, where

$$
\mathcal{P}_{3}=\mathscr{P}_{A} \cdot \mathscr{P}_{B} \cdot \mathscr{P}_{C}
$$

and

$\mathcal{P}_{E \mid 3}\left(\mathcal{B}_{t}\right)=\mathbb{P}\left\{\mathcal{B}_{c} \cap \mathcal{B}_{t}^{C} \mid p_{e}=0\right\}+\mathbb{P}\left\{\mathcal{B}_{c} \cap \mathcal{B}_{t}^{C} \mid 0<p_{e} \leq 0.5\right\}$.

In the case $\mathcal{B}_{t}=\mathcal{B}_{t_{1}}, \mathcal{P}_{E \mid 3}\left(\mathcal{B}_{t_{1}}\right)$ is given by (28), whereas in 


$$
\begin{aligned}
\mathcal{P}_{E \mid 2} & =\mathbb{P}\left\{\mathcal{A}_{4} \mid p_{e}=0\right\}+\mathbb{P}\left\{\mathcal{A}_{4} \mid 0<p_{e} \leq 0.5\right\}, \\
& =\frac{\mathbb{P}^{\prime}\left\{\mathcal{A}_{4_{a}} \mid p_{e}=0\right\}+\mathbb{P}^{\prime}\left\{\mathcal{A}_{4_{b}} \mid p_{e}=0\right\}+\mathbb{P}^{\prime}\left\{\mathcal{A}_{4_{a}} \mid 0<p_{e} \leq 0.5\right\}+\mathbb{P}^{\prime}\left\{\mathcal{A}_{4_{b}} \mid 0<p_{e} \leq 0.5\right\}}{\mathbb{P}\left(\mathcal{A}_{4} \cup \mathcal{A}_{5}\right)},
\end{aligned}
$$

with $\mathbb{P}\left\{\star \mid p_{e}=0\right\}$ and $\mathbb{P}\left\{\star \mid 0<p_{e} \leq 0.5\right\}$ denoting the probability that the corresponding rate vector, $\left(R_{A}, R_{B}\right)$ for $M 2$ and $\left(R_{A}, R_{B}, R_{C}\right)$ for $M 3$, fall in region $\star$ given the cases $p_{e}=0$ and $0<p_{e} \leq 0.5$, respectively. $\mathbb{P}^{\prime}(\bullet)$ is the unnormalized value of $\mathbb{P}(\bullet)$ given the fact that $\mathbb{P}\left[\left(R_{A}, R_{B}\right) \in \mathcal{A}_{4} \cup \mathcal{A}_{5}\right]=1$.

$$
\begin{aligned}
\mathcal{P}_{E \mid 3}\left(\mathcal{B}_{t_{1}}\right) & =\mathbb{P}\left\{\mathcal{B}_{c} \mid p_{e}=0\right\}-\mathbb{P}\left\{\mathcal{B}_{t_{1}} \mid p_{e}=0\right\}+\mathbb{P}\left\{\mathcal{B}_{c} \mid 0<p_{e} \leq 0.5\right\}-\mathbb{P}\left\{\mathcal{B}_{t_{1}} \mid 0<p_{e} \leq 0.5\right\}, \\
& =\frac{\mathbb{P}^{\prime}\left\{\mathcal{B}_{c} \mid p_{e}=0\right\}-\mathbb{P}^{\prime}\left\{\mathcal{B}_{t_{1}} \mid p_{e}=0\right\}+\mathbb{P}^{\prime}\left\{\mathcal{B}_{c} \mid 0<p_{e} \leq 0.5\right\}-\mathbb{P}^{\prime}\left\{\mathcal{B}_{t_{1}} \mid 0<p_{e} \leq 0.5\right\}}{\mathbb{P}\left(\mathcal{B}_{c}\right)}
\end{aligned}
$$

the case of $\mathcal{B}_{t}=\mathcal{B}_{t_{2}}$,

$$
\begin{aligned}
\mathcal{P}_{E \mid 3}\left(\mathcal{B}_{t_{2}}\right) & =\mathbb{P}\left\{\mathcal{B}_{t_{2}}^{C} \mid p_{e}=0\right\}+\mathbb{P}\left\{\mathcal{B}_{t_{2}}^{C} \mid 0<p_{e} \leq 0.5\right\}, \\
& =\frac{\mathbb{P}^{\prime}\left\{\mathcal{B}_{t_{2}}^{C} \mid p_{e}=0\right\}+\mathbb{P}^{\prime}\left\{\mathcal{B}_{t_{2}}^{C} \mid 0<p_{e} \leq 0.5\right\}}{\mathbb{P}\left(\mathcal{B}_{C}\right)} .
\end{aligned}
$$

The normalization factor $\mathbb{P}\left(\mathcal{B}_{c}\right)$ that appears in common in the denominator of (28) and (29) is defined by

$$
\begin{aligned}
\mathbb{P}\left(\mathcal{B}_{C}\right)= & \int_{\Phi_{A}^{-1}(0)}^{\Phi_{A}^{-1}(1)} \int_{\Phi_{B}^{-1}(0)}^{\Phi_{B}^{-1}(1)} \int_{\Phi_{C}^{-1}(0)}^{\Phi_{C}^{-1}(1)} p\left(\gamma_{A}\right) p\left(\gamma_{B}\right) p\left(\gamma_{C}\right) d_{\gamma_{A}} d_{\gamma_{B}} d_{\gamma_{C}} \\
= & {\left[1-\exp \left(-\frac{\Phi_{A}^{-1}(1)}{\Gamma_{A}}\right)\right]\left[1-\exp \left(-\frac{\Phi_{B}^{-1}(1)}{\Gamma_{B}}\right)\right] } \\
& {\left[1-\exp \left(-\frac{\Phi_{C}^{-1}(1)}{\Gamma_{C}}\right)\right] . }
\end{aligned}
$$

The other probability expressions in (28) are given by the equations (45)-(48) in Appendix D.

\section{Numerical Analyses}

This section presents numerical results of the upper bound of the outage probability bound for $M 2$ and $M 3$, given particular $\rho_{z}$ and $Q_{n}$ values. With any values of $\rho_{z}, M 2$ can always achieve diversity order two, while, M3 can achieve diversity order four if the information correlation is close to one; otherwise, M3 can always achieve the diversity order three. To confirm this fact, we perform a series of computer simulations for $10^{6}$ channel realizations and $\rho_{A B}=\rho_{B C}=$ $\{0,0.5,0.8,0.98,1\}$, the results of which are shown in Fig. 5. The results of a series of Monte Carlo simulations and theoretical calculations are shown in the figure. It is found that they are consistent each other.

With $M 2$, the high packet correlations provide slightly better performance at low average SNR regime. However, no significant improvement can be achieved with any packet correlation value at high average SNR regime. Nevertheless, the diversity order is two for all the cases. It is understandable by analyzing the $M 2$ inadmissible rate region, where the shape of the case $\mathbb{P}\left[\left(R_{A}, R_{B}\right) \in \mathcal{A}_{5}\right]=0$ leads the result of the integral calculation inversely proportional to $\left(\Gamma_{n}\right)^{2}$. Furthermore, this case highly likely occurs at high average SNR value range where $p_{e} \approx 0$ and hence $R_{A}+R_{B} \geq 1+H_{b}\left(p_{A B}\right)-\theta_{2} \approx 1$.

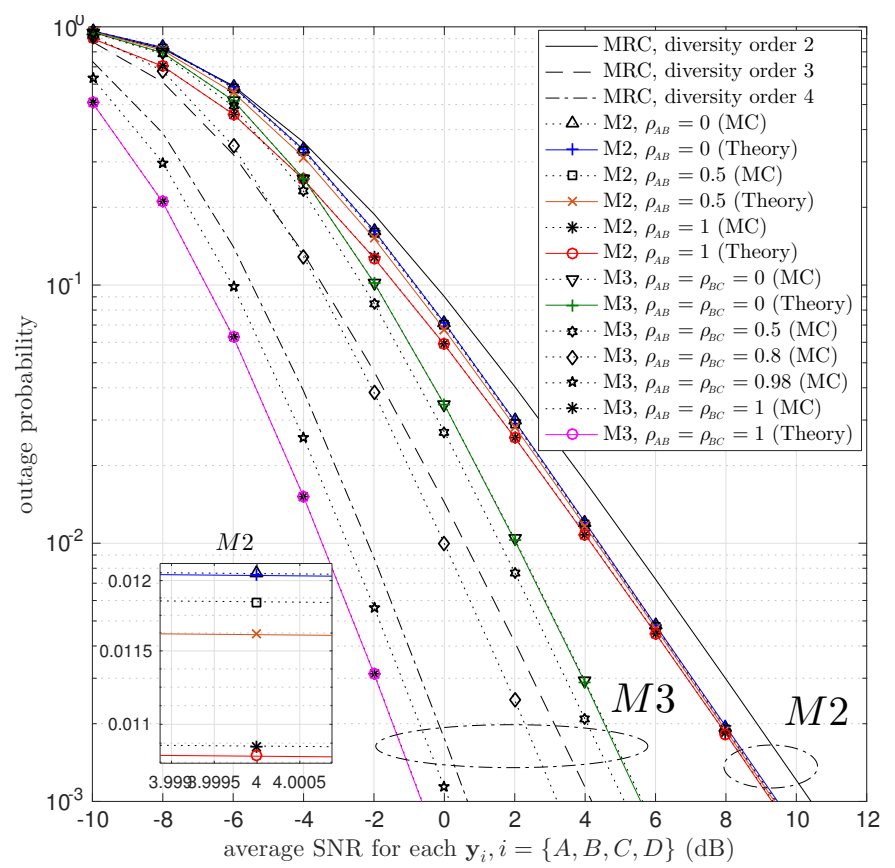

Fig. 5: Upper bound of the outage probabilities of feedbackassisted correlated packet transmission with $M 2$ and $M 3$ for equal transmit power and $Q_{n}=0.5$.

With $M 3$, the performance is parameterized with $\rho_{A B}$ and $\rho_{B C}$. It is easy to find that $p_{A B C} \approx 0.5$, corresponding to $\rho_{A B C} \approx 0$, for any values of the pair $\rho_{A B}$ and $\rho_{B C}$. Since the integral boundary is complex, depending on $p_{A B}$ and $p_{B C}$, we calculated theoretically the outage probability only for the simplest case which are $p_{A B}=p_{B C}=0.5$ corresponding to $\rho_{A B}=\rho_{B C}=0$, and $p_{A B}=p_{B C}=0$ to $\rho_{A B}=\rho_{B C}=1$; and for the other cases, we calculated the outage probability by Monte Carlo simulations.

It is found from Fig. 5 that with $M 3$, the Monte Carlo simulation and theoretical results are consistent for $\rho_{A B}=$ $\rho_{B C}=0$ and $\rho_{A B}=\rho_{B C}=1$, and for the other cases, the diversity order indicated by the decay of the curves are the same. It is found that except for the information correlation being very close to one, the diversity order three can always be achieved. With $\rho_{A B}=\rho_{B C}=0.8$, we can achieve roughly $2 \mathrm{~dB}$ 
improvement at outage probability of $10^{-3}$, compared to that with $\rho_{A B}=\rho_{B C}=0$, but, again, it should be emphasized that the diversity order for all the cases is the same. However, in the case the information correlation is very close to one, $M 3$ can achieve the diversity order four. This is reasonable because, with the correlation close to one, all the packets including the helper are almost the same, and hence in this case almost the same packet is transmitted four times.

Fig. 6 and Fig. 7 show the performance of $M 2$ and $M 3$ for various $Q_{n}$ and transmit power allocations to the information and the helper packets, respectively. Note that low signaling spectrum efficiency $Q_{n}$ corresponds to large redundancy while modulation order fixed, or low modulation order while the channel coding redundancy fixed. ${ }^{7}$ It is found in Fig. 6 that in general, low $Q_{n}$ slightly improves the performance of $M 2$. Specifically, the impact of decreasing $Q_{n}$ for the information packet improves the outage performance more significantly than decreasing that of the helper packet. Also, it is found from Fig. 7 that increasing the transmit power of the information packet improves the performance more significantly than that of the helper packet. Likewise, the same tendency also can be found for $M 3$ case as shown in Fig. 7. In summary, (a) using smaller signaling spectrum efficiency can achieve smaller outage probability, and (b) increasing the transmit power of the information packet is more effective in reducing the outage probability than increasing that of the helper packet. The improvement of (a) and (b) is in the form of the parallel shift of the outage curve.

\section{Generalization}

In this Section, for the sake of clarity, we replace the alphabets in the subscripts of the rates $R$ and bit-flipping variables $p$, with numbers, e.g., $R_{A} \rightarrow R_{1}, R_{B} \rightarrow R_{2}, p_{A B} \rightarrow$ $p_{12}, p_{A B C} \rightarrow p_{123}, p_{A B C D \ldots} \rightarrow p_{1234 \ldots .}$. We also use subscript help instead of $D$ to indicate the helper packet possession.

We showed in Section III that with $M$ NACK-ed packets, the largest inadmissible rate region is given by $\mathfrak{S}=$ $\left\{\left(R_{1}, R_{2}, \cdots, R_{M}\right) \mid 0 \leq R_{i}<1, i=\{1,2, \cdots, M\}\right\}$. With the calculation as in Section IV, the dimension of $\mathfrak{S}$ determines the $M$ order diversity. The physical meaning of such system is that the receiver always combines $M$ NACKed packets and these packets are unrecovered at first; however, only by utilizing the source correlation and/or helper packet, there is a chance to recover all $M$ packets.

With full information correlation, however, it is noticeable that bitwise XOR operation makes the helper the same as all NACK-ed packets if $M$ is odd, and hence $M+1$ order diversity can be achieved. On the other hand, the helper packet is always the binary zeros if $M$ is even, and hence with the full correlation, the bit pattern in the helper packet is not uniquely corresponding to the information packet. Therefore, only $M$ order diversity can be achieved. For this reason, it is still interesting to see how odd number of $M$ can achieve beyond the diversity order $M$ by mathematical formulas using the theorem for multiple source coding with a helper.

\footnotetext{
${ }^{7}$ See footnote 4.
}

In Section III, we showed that the cut size of the inadmissible rate region determines additional gain. The larger the area/volume of the cut inadmissible rate region corresponds to the lower the outage probability. Let $\mathfrak{R}$ be an area or a volume containing the rates, as $\mathfrak{R}=\left\{\left(R_{1}, R_{2}, \cdots, R_{M}\right) \in\right.$ $\left.\mathbb{R}^{+} \mid \sum_{i=1}^{M} R_{i}<H\left(\boldsymbol{u}_{1}, \boldsymbol{u}_{2}, \cdots, \boldsymbol{u}_{M} \mid \hat{\boldsymbol{u}}_{\text {help }}\right)\right\}$, the area/volume of the cut inadmissible rate region, denoted by $\mathfrak{C}$, is then given by

$$
\mathfrak{C}=\mathfrak{S}-\mathfrak{R}, \forall \mathfrak{R} \subseteq \mathfrak{S}
$$

It can be seen that $\mathfrak{C}$ is the significant factor that determines the sum-rates in $\mathfrak{R}$, as

$$
\begin{aligned}
\sum_{i=1}^{M} R_{i}< & H\left(\boldsymbol{u}_{1}, \boldsymbol{u}_{2}, \cdots, \boldsymbol{u}_{M} \mid \hat{\boldsymbol{u}}_{\text {help }}\right) \\
= & H\left(\boldsymbol{u}_{1}\right)+\sum_{j=2}^{M} H\left(\boldsymbol{u}_{j} \mid \boldsymbol{u}_{1}, \boldsymbol{u}_{2}, \cdots, \boldsymbol{u}_{j-1}\right) \\
& +H\left(\hat{\boldsymbol{u}}_{\text {help }} \mid \boldsymbol{u}_{1}, \boldsymbol{u}_{2}, \cdots, \boldsymbol{u}_{M}\right)-H\left(\hat{\boldsymbol{u}}_{\text {help }}\right) \\
= & 1+\sum_{j=2}^{M} H_{b}\left(p_{j-1, j}\right)+H_{b}\left(p_{e}\right)-H_{b}\left(p_{1 \cdots M} * p_{e}\right),
\end{aligned}
$$

where $H_{b}\left(p_{j-1, j}\right)$ in (32) is because of the source correlation and $p_{j-1, j}=\mathbb{P}\left(\boldsymbol{u}_{j-1} \oplus \boldsymbol{u}_{j}=1\right)$. We can find that $\sum_{i=1}^{M} R_{i}<$ $M-1$ if the sources are uncorrelated and the helper is error free. In this case $\mathfrak{C}=0$, and hence there is no additional gain.

On the other hand, with full information correlation, $p_{1 \cdots M}$ is respectively being 0 or 0.5 if $M$ is even or odd. ${ }^{8}$ Therefore, $\sum_{i=1}^{M} R_{i}<1$ if $M$ is even and $\sum_{i=1}^{M} R_{i}<H_{b}\left(p_{e}\right)$ if $M$ is odd, which are corresponding to $\mathfrak{C}=0$ and $\mathfrak{C} \geq 0$, respectively.

It can be seen that the equality $\mathfrak{C}=0$ with $M$ being odd holds only when the bit error probability of the helper packet is 0.5 . This case is equivalent to the case with $M$ being even, which is the bit pattern in the helper packet is not uniquely corresponding to the information packet. Hence, only $M$-th order diversity can be achieved, even with large information packet correlation. Therefore, no additional diversity gain can be achieved with $M$ being even, on the contrary, $(M+1)$-th order diversity can be achieved with very large information packet correlation with $M$ being odd.

\section{CONCLUSION}

We analyzed the relationship among outage probability, achievable diversity gain, and source information correlation for an $M$-in-1 helper transmission system. We derived a fully mathematical expression for the relationship only with $M=\{2,3\}$ as the initial investigation. Furthermore, this paper fully analyzed for any value of $M$ the relationship between the diversity order and the rate sum for the case where all packets are fully correlated as an extreme case.

We have analyzed the inadmissible rate region with $M=$ $\{2,3\}$. The helper packet is formed by taking bitwise binary

\footnotetext{
${ }^{8}$ This is because the definition of $p_{1} \cdots M=\mathbb{P}\left(u_{1} \oplus u_{2} \oplus \cdots \oplus u_{M}=1\right)$.
} 


$$
\begin{aligned}
\square & =R_{B}=1, R_{D}=1 \\
R_{A} & =R_{B}=1, R_{D}=0.5 \\
\square & R_{A}=R_{B}=0.5, R_{D}=1 \\
\square R_{A} & =R_{B}=0.5, R_{D}=0.5
\end{aligned}
$$

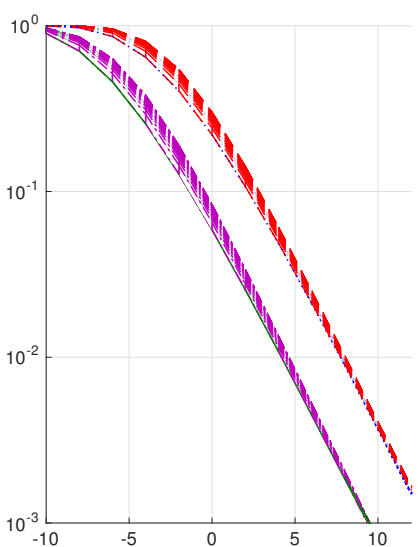

Average SNR for each $\mathbf{y}_{i}(\mathrm{~dB})$ $i=\{A, B\}$

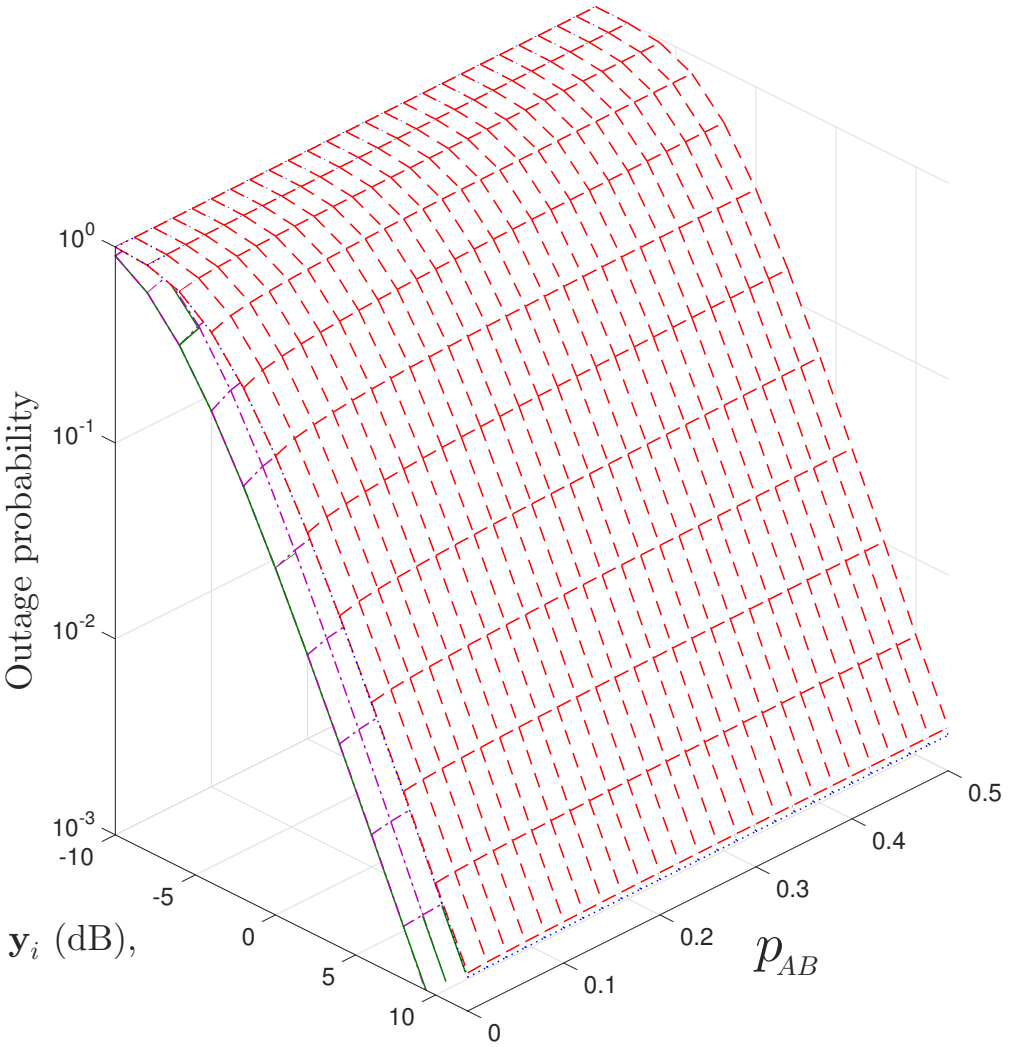

(a) $\Gamma_{D}=\Gamma_{\{A, B\}}$.

$$
\begin{aligned}
\square & =R_{B}=1, R_{D}=1 \\
R_{A} & =R_{B}=1, R_{D}=0.5 \\
R_{A} & =R_{B}=0.5, R_{D}=1 \\
\square R_{A} & =R_{B}=0.5, R_{D}=0.5
\end{aligned}
$$

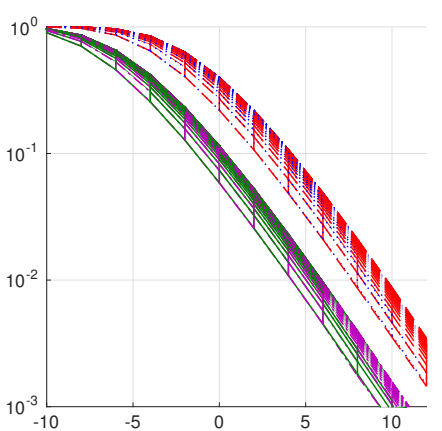

Average SNR for each $\mathbf{y}_{i}(\mathrm{~dB})$, $i=\{A, B\}$

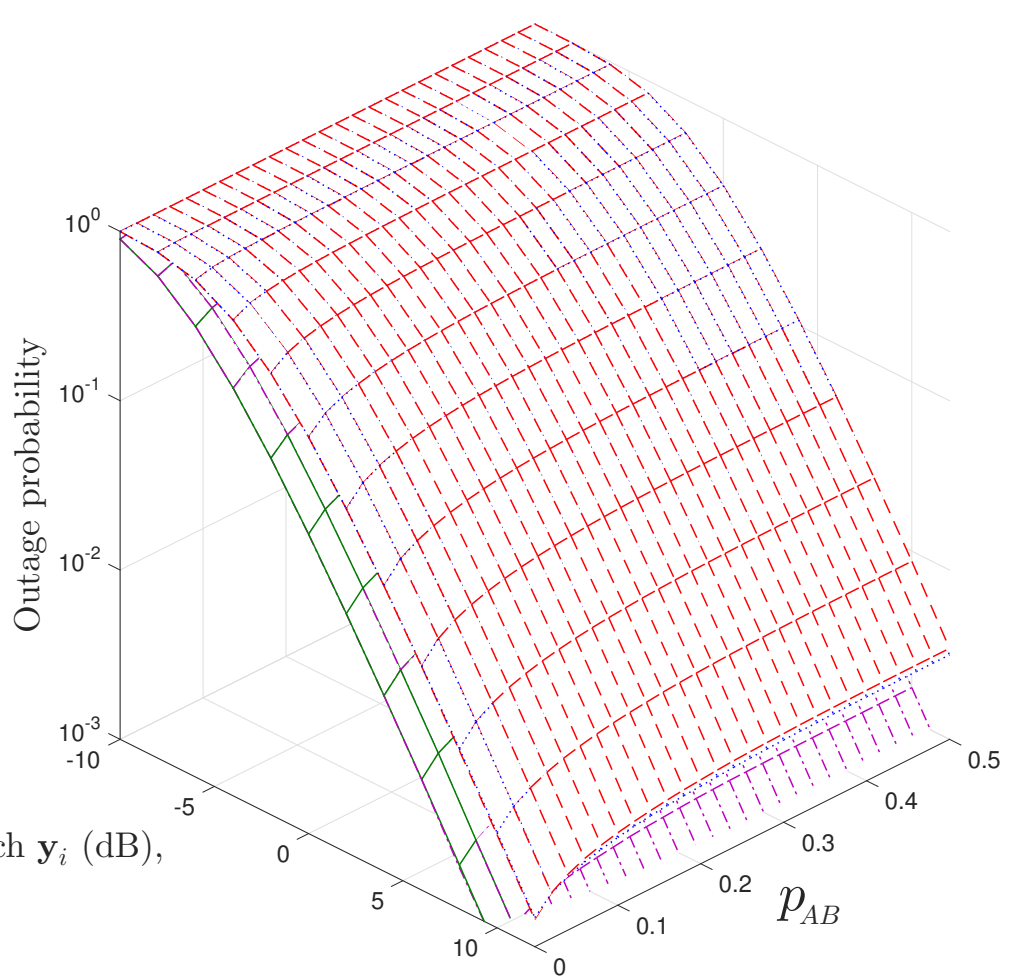

(b) $\Gamma_{D}=\Gamma_{\{A, B\}}-20 d B$.

Fig. 6: Upper bound of the outage probability of $M 2$ for unequal transmit power for the information and helper packets. 


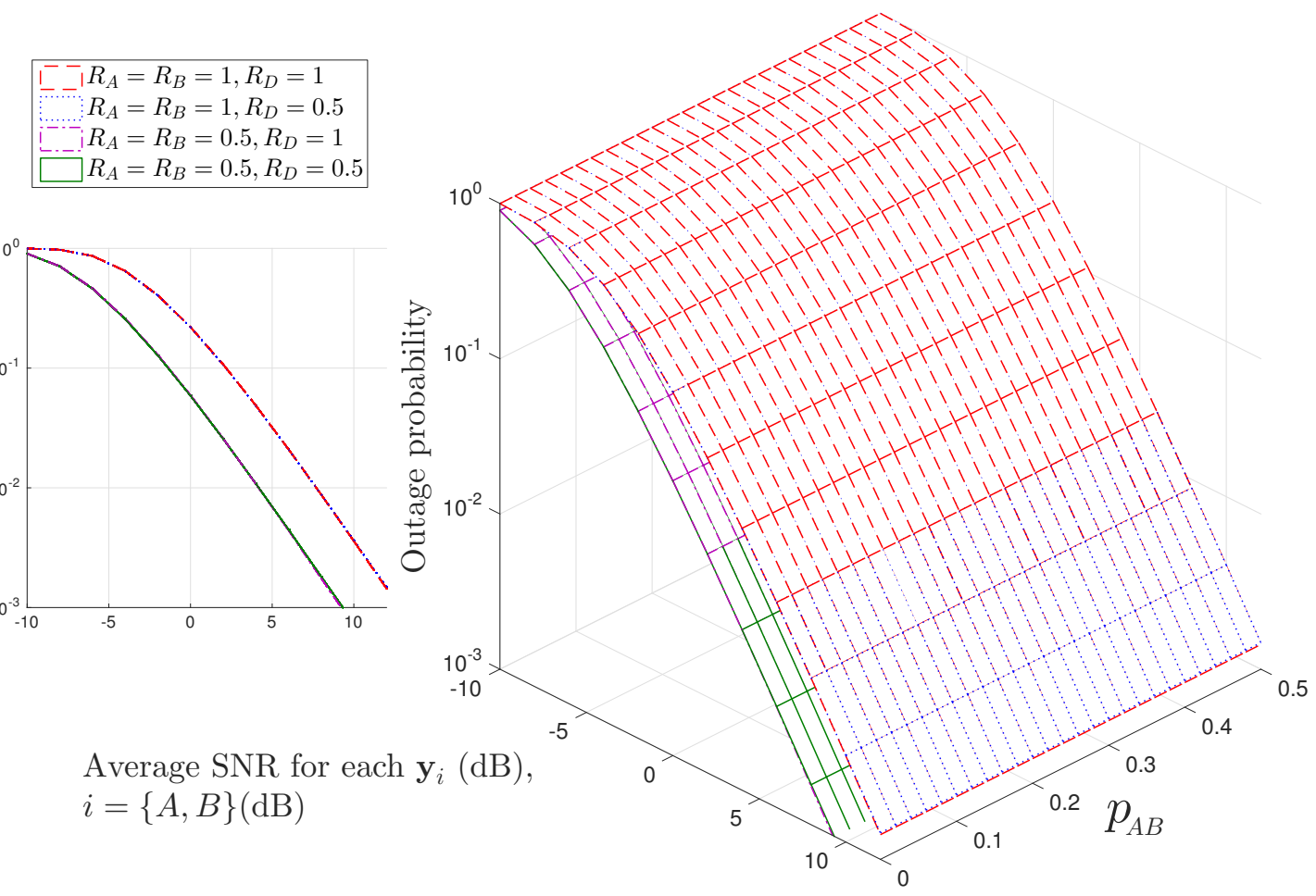

(c) $\Gamma_{D}=\Gamma_{\{A, B\}}+20 d B$.

XOR over the information packets identified as erroneously received by the decoder, of which event the transmitter has been notified of via the feedback channel. We used the theorem given in [22, Theorem 10.4] to derive the inadmissible rate region. We then derived the upper bound of the outage probability of the system over block Rayleigh fading channels. By the definition of the outage, it is noticeable that at least $M$-th order diversity can always be achieved regardless of the information correlation. We then proved that there is no additional diversity order that can be achieved with $M$ being even, but $(M+1)$-th order diversity can be achieved with $M$ being an odd number, especially when the information correlation is close to one.

The results of this work are relevant and important for designing future wireless networks having massive terminals which are transmitting information generated or sensed from the same source and hence correlated. The practical applications include, but not limited to, infrastructure-to-vehicle (I2V) communication, wireless mobile sensor networks, and wireless Internet-of-Things (IoT).

Identifying practical joint decoding algorithms that can achieve the performance supported by the theoretical analyses provided in this paper is left as a future study. Also, deriving explicit mathematical formulas representing the relationship between outage probability, source information correlation, diversity order, and required average SNR for $M>3$ is left as a very interesting topic.

\section{APPENDIX A}

DERIVATION OF OUTAGE FOR $M=2$ (WITHOUT FEEDBACK)

The admissible rate region of $M 2$ without feedback is given by [22]

$$
\begin{aligned}
R_{A} & \geq H\left(\boldsymbol{u}_{A} \mid \boldsymbol{u}_{B}, \hat{\boldsymbol{u}}_{D}\right), \\
R_{B} & \geq H\left(\boldsymbol{u}_{B} \mid \boldsymbol{u}_{A}, \hat{\boldsymbol{u}}_{D}\right), \\
R_{A}+R_{B} & \geq H\left(\boldsymbol{u}_{A}, \boldsymbol{u}_{B} \mid \hat{\boldsymbol{u}}_{D}\right), \\
R_{D} & \geq I\left(\boldsymbol{u}_{D} ; \hat{\boldsymbol{u}}_{D}\right) .
\end{aligned}
$$

The mutual information in (33d) can be further derived as

$$
\begin{aligned}
I\left(\boldsymbol{u}_{D} ; \hat{\boldsymbol{u}}_{D}\right) & =H\left(\hat{\boldsymbol{u}}_{D}\right)-H\left(\hat{\boldsymbol{u}}_{D} \mid \boldsymbol{u}_{D}\right), \\
& =H\left(\boldsymbol{\nu}_{A B} \oplus \boldsymbol{\nu}_{e}\right)-H_{b}\left(p_{e}\right), \\
& =H_{b}\left(p_{A B} * p_{e}\right)-H_{b}\left(p_{e}\right), \\
& \triangleq \theta_{2} .
\end{aligned}
$$

With the result of (34), the conditional entropy in (33a) can be modified as

$$
\begin{aligned}
H\left(\boldsymbol{u}_{A} \mid \boldsymbol{u}_{B}, \hat{\boldsymbol{u}}_{D}\right)= & H\left(\boldsymbol{u}_{B}\right)+H\left(\boldsymbol{u}_{A} \mid \boldsymbol{u}_{B}\right)+H\left(\hat{\boldsymbol{u}}_{D} \mid \boldsymbol{u}_{A}, \boldsymbol{u}_{B}\right) \\
& -H\left(\boldsymbol{u}_{B}, \hat{\boldsymbol{u}}_{D}\right), \\
= & H\left(\boldsymbol{u}_{B}\right)+H_{b}\left(p_{A B}\right)+H\left(\boldsymbol{u}_{A} \oplus \boldsymbol{u}_{B} \oplus \boldsymbol{\nu}_{e} \mid \boldsymbol{u}_{A}, \boldsymbol{u}_{B}\right) \\
& -\left[H\left(\boldsymbol{u}_{B}\right)+H\left(\hat{\boldsymbol{u}}_{D} \mid \boldsymbol{u}_{B}\right)\right], \\
= & H_{b}\left(p_{A B}\right)+H_{b}\left(p_{e}\right)-H\left(\boldsymbol{u}_{A} \oplus \boldsymbol{u}_{B} \oplus \boldsymbol{\nu}_{e} \mid \boldsymbol{u}_{B}\right), \\
= & H_{b}\left(p_{A B}\right)+H_{b}\left(p_{e}\right)-H\left(\boldsymbol{u}_{B} \oplus \boldsymbol{\nu}_{A B} \oplus \boldsymbol{u}_{B} \oplus \boldsymbol{\nu}_{e} \mid \boldsymbol{u}_{B}\right), \\
= & H_{b}\left(p_{A B}\right)+H_{b}\left(p_{e}\right)-H\left(\boldsymbol{\nu}_{A B} \oplus \boldsymbol{\nu}_{e}\right), \\
= & H_{b}\left(p_{A B}\right)-\theta_{2} .
\end{aligned}
$$




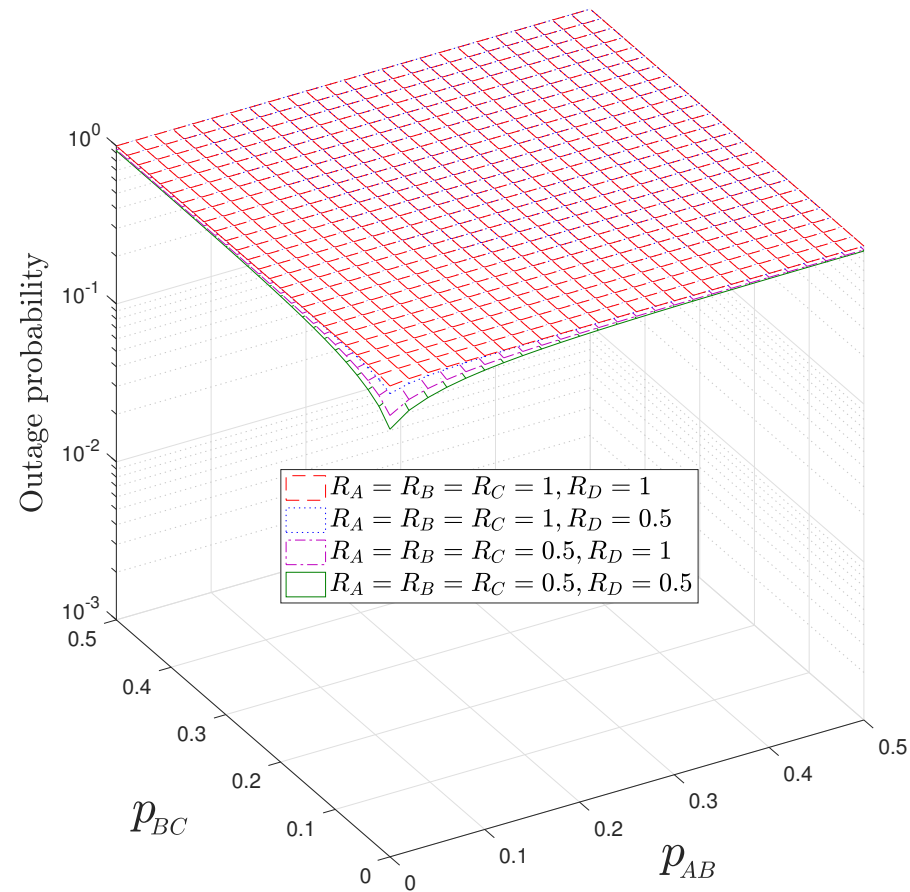

(a) $\Gamma_{D}=\Gamma_{\{A, B, C\}}=-10 \mathrm{~dB}$.

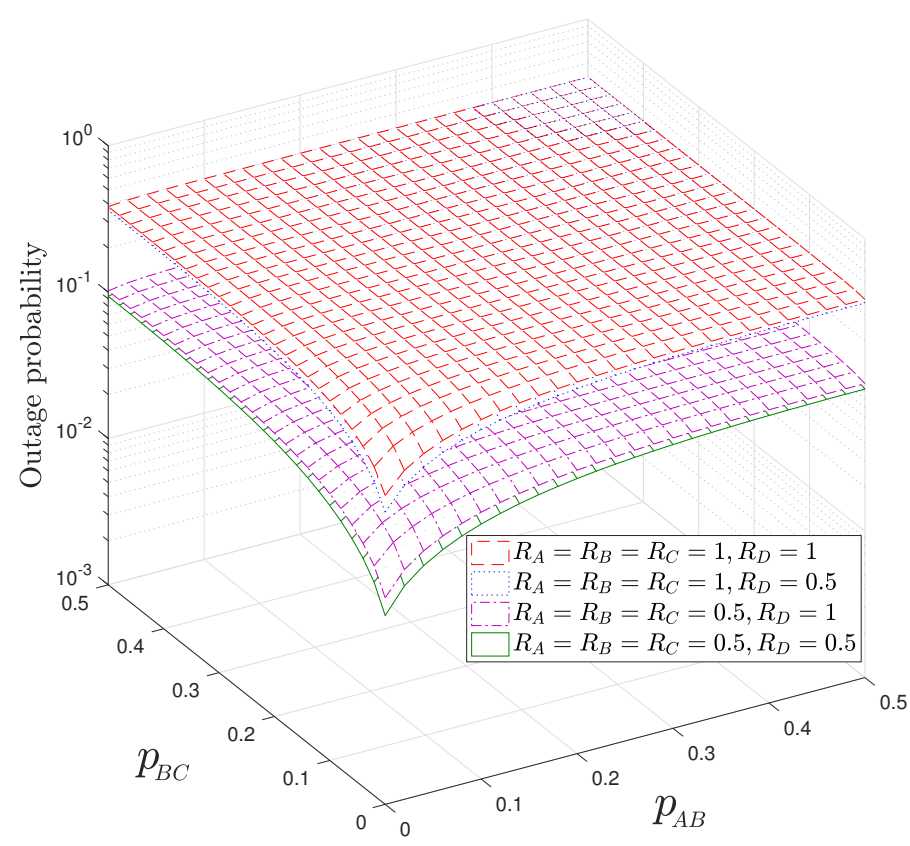

(c) $\Gamma_{D}=-10 d B, \Gamma_{\{A, B, C\}}=0 d B$.

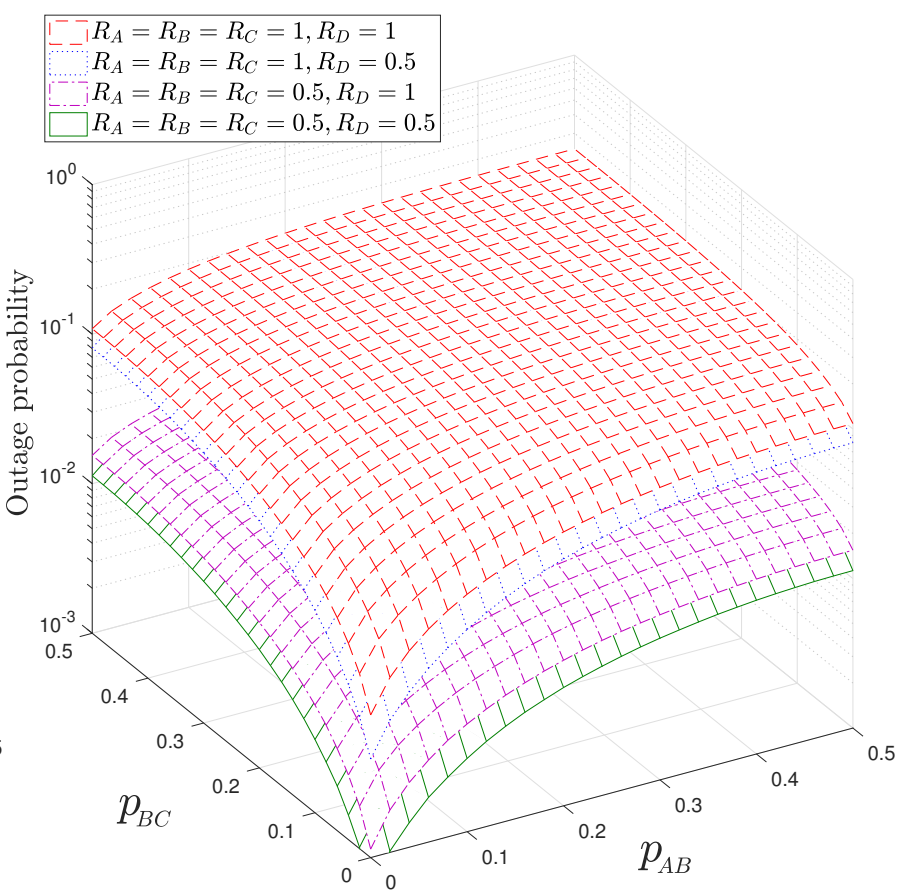

(b) $\Gamma_{D}=\Gamma_{\{A, B, C\}}=0 d B$.

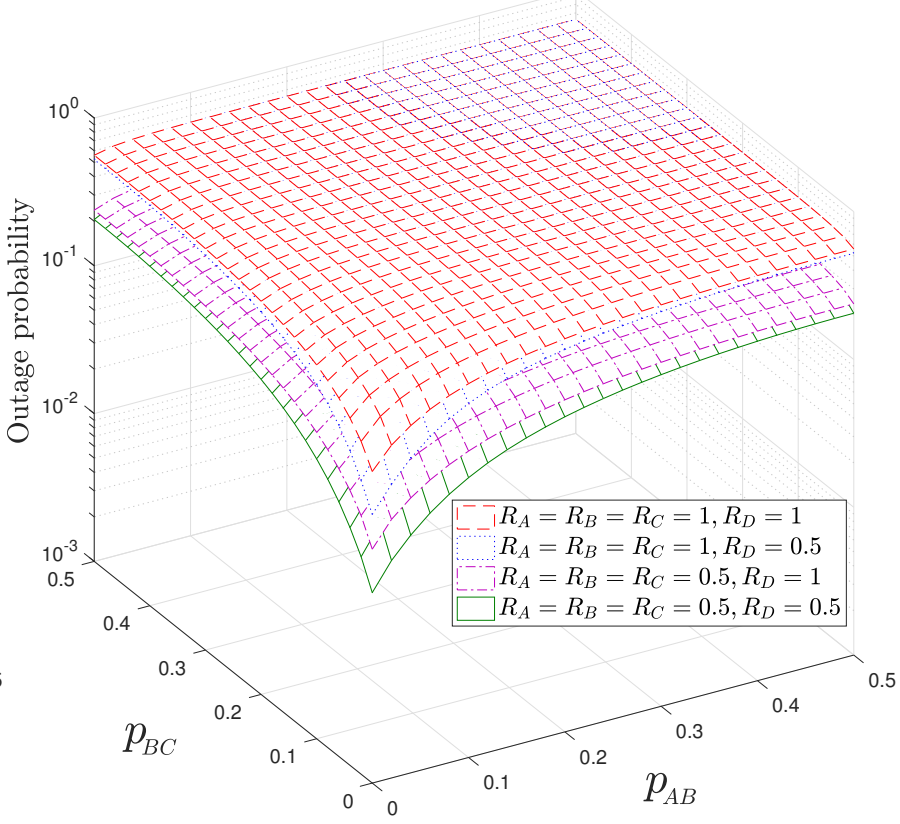

(d) $\Gamma_{D}=0 d B, \Gamma_{\{A, B, C\}}=-10 d B$.

Fig. 7: Upper bound of the outage probability of $M 3$ for various transmit power settings. 
Likewise, the conditional entropy in (33b) can be modified as

$$
H\left(\boldsymbol{u}_{B} \mid \boldsymbol{u}_{A}, \hat{\boldsymbol{u}}_{D}\right)=H_{b}\left(p_{A B}\right)-\theta_{2} .
$$

Eventually, the conditional entropy in (33c) can be modified as

$$
H\left(\boldsymbol{u}_{A}, \boldsymbol{u}_{B} \mid \hat{\boldsymbol{u}}_{D}\right)=1+H_{b}\left(p_{A B}\right)-\theta_{2} .
$$

Therefore, based on the results of (34)-(37), inequalities (33a)-(33d) can be rewritten as follows.

$$
\begin{aligned}
R_{A} \geq H_{b}\left(p_{A B}\right)-\theta_{2}, \\
R_{B} \geq H_{b}\left(p_{A B}\right)-\theta_{2}, \\
R_{A}+R_{B} \geq 1+H_{b}\left(p_{A B}\right)-\theta_{2}, \\
R_{D} \geq \theta_{2} .
\end{aligned}
$$

\section{APPENDIX B}

DERViation of OUTAGE FOR $M=3$ (Without

\section{FEEDBACK)}

The admissible rate region of $M 3$ without feedback is given by [22]

$$
\begin{aligned}
R_{A} \geq H\left(\boldsymbol{u}_{A} \mid \boldsymbol{u}_{B}, \boldsymbol{u}_{C}, \hat{\boldsymbol{u}}_{D}\right), \\
R_{B} \geq H\left(\boldsymbol{u}_{B} \mid \boldsymbol{u}_{A}, \boldsymbol{u}_{C}, \hat{\boldsymbol{u}}_{D}\right), \\
R_{C} \geq H\left(\boldsymbol{u}_{C} \mid \boldsymbol{u}_{A}, \boldsymbol{u}_{B}, \hat{\boldsymbol{u}}_{D}\right), \\
R_{A}+R_{B} \geq H\left(\boldsymbol{u}_{A}, \boldsymbol{u}_{B} \mid \boldsymbol{u}_{C}, \hat{\boldsymbol{u}}_{D}\right), \\
R_{A}+R_{C} \geq H\left(\boldsymbol{u}_{A}, \boldsymbol{u}_{C} \mid \boldsymbol{u}_{B}, \hat{\boldsymbol{u}}_{D}\right), \\
R_{B}+R_{C} \geq H\left(\boldsymbol{u}_{B}, \boldsymbol{u}_{C} \mid \boldsymbol{u}_{A}, \hat{\boldsymbol{u}}_{D}\right), \\
R_{A}+R_{B}+R_{C} \geq H\left(\boldsymbol{u}_{A}, \boldsymbol{u}_{B}, \boldsymbol{u}_{C} \mid \hat{\boldsymbol{u}}_{D}\right), \\
R_{D} \geq I\left(\boldsymbol{u}_{D} ; \hat{\boldsymbol{u}}_{D}\right) .
\end{aligned}
$$

With the same method that is used in Appendix A, the inequalities (39a)-(39h) can be rewritten as follows.

$$
\begin{gathered}
R_{A} \geq H_{b}\left(p_{A B}\right)-\theta_{3}, \\
R_{B} \geq H_{b}\left(p_{A B}\right)-\theta_{3}, \\
R_{C} \geq H_{b}\left(p_{B C}\right)-\theta_{3}, \\
R_{A}+R_{B} \geq H_{b}\left(p_{A B}\right)+H_{b}\left(p_{B C}\right)-\theta_{3}, \\
R_{A}+R_{C} \geq H_{b}\left(p_{A B}\right)+H_{b}\left(p_{B C}\right)-\theta_{3}, \\
R_{B}+R_{C} \geq H_{b}\left(p_{A B}\right)+H_{b}\left(p_{B C}\right)-\theta_{3}, \\
R_{A}+R_{B}+R_{C} \geq 1+H_{b}\left(p_{A B}\right)+H_{b}\left(p_{B C}\right)-\theta_{3}, \\
R_{D} \geq \theta_{3},
\end{gathered}
$$

where $\theta_{3}=H_{b}\left(p_{A B C} * p_{e}\right)-H_{b}\left(p_{e}\right)$.

$$
\begin{gathered}
\text { Appendix C } \\
\text { Supporting EQuATIOns For OUtaGe Probability } \\
\text { Derivation of } M 2 \\
\mathbb{P}^{\prime}\left\{\mathcal{A}_{4_{a}} \mid p_{e}=0\right\}=\int_{\Phi_{D}^{-1}\left[H_{b}\left(p_{A B}\right)\right] \Phi_{A}^{-1}(0)}^{\Phi_{D}^{-1}(\infty)} \int_{\Phi_{B}^{-1}(0)}^{\Phi_{A}^{-1}(0) \Phi_{B}^{-1}(1)} \\
=0
\end{gathered}
$$

$$
\begin{aligned}
& \mathbb{P}^{\prime}\left\{\mathcal{A}_{4_{b}} \mid p_{e}=0\right\} \\
& \Phi_{D}^{-1}(\infty) \quad \Phi_{A}^{-1}(1) \Phi_{B}^{-1}\left[1-\Phi_{A}\left(\gamma_{A}\right)\right] \\
& =\int_{\Phi_{D}^{-1}\left[H_{b}\left(p_{A B}\right)\right]}^{D} \int_{\Phi_{A}^{-1}(0)}^{A} \int_{\Phi_{B}^{-1}(0)} p\left(\gamma_{B}\right) p\left(\gamma_{A}\right) p\left(\gamma_{D}\right) d \gamma_{B} d_{\gamma_{A}} d \gamma_{D} \\
& =\frac{1}{\Gamma_{A}} \exp \left(-\frac{\Phi_{D}^{-1}\left[H_{b}\left(p_{A B}\right)\right]}{\Gamma_{D}}\right) \int_{\Phi_{A}^{-1}(0)}^{\Phi_{A}^{-1}(1)} \exp \left(-\frac{\gamma_{A}}{\Gamma_{A}}\right) \\
& {\left[1-\exp \left(-\frac{\Phi_{B}^{-1}\left[1-\Phi_{A}\left(\gamma_{A}\right)\right]}{\Gamma_{B}}\right)\right] d_{\gamma_{A}} .} \\
& \mathbb{P}^{\prime}\left\{\mathcal{A}_{4_{a}} \mid 0<p_{e} \leq 0.5\right\} \\
& \Phi_{D}^{-1}\left[H_{b}\left(p_{A B}\right)\right] \Phi_{A}^{-1}\left[\Psi\left(\gamma_{D}\right)\right] \Phi_{B}^{-1}(1) \\
& =\int_{\Phi_{D}^{-1}(0)} \int_{\Phi_{A}^{-1}(0)} \int_{\Phi_{B}^{-1}(0)} p\left(\gamma_{B}\right) p\left(\gamma_{A}\right) p\left(\gamma_{D}\right) d \gamma_{B} d \gamma_{A} d \gamma_{D} \\
& =\frac{1}{\Gamma_{D}}\left[1-\exp \left(-\frac{\Phi_{B}^{-1}(1)}{\Gamma_{B}}\right)\right] \int_{\Phi_{D}^{-1}(0)}^{\Phi_{D}^{-1}\left[H_{b}\left(p_{A B}\right)\right]} \exp \left(-\frac{\gamma_{D}}{\Gamma_{D}}\right) \\
& {\left[1-\exp \left(-\frac{\Phi_{A}^{-1}\left[\Psi\left(\gamma_{D}\right)\right]}{\Gamma_{A}}\right)\right] d_{\gamma_{D}}} \\
& \mathbb{P}^{\prime}\left\{\mathcal{A}_{4_{b}} \mid 0<p_{e} \leq 0.5\right\} \\
& \Phi_{D}^{-1}\left[H_{b}\left(p_{A B}\right)\right] \Phi_{A}^{-1}(1) \Phi_{B}^{-1}\left[\Psi\left(\gamma_{A}, \gamma_{D}\right)\right] \\
& =\int_{\Phi_{D}^{-1}(0)} \int_{\Phi_{A}^{-1}\left[\Psi\left(\gamma_{D}\right)\right]} \int_{\Phi_{B}^{-1}(0)} p\left(\gamma_{B}\right) p\left(\gamma_{A}\right) p\left(\gamma_{D}\right) d_{\gamma_{B}} d \gamma_{A} d \gamma_{D} \\
& \Phi_{D}^{-1}\left[H_{b}\left(p_{A B}\right)\right] \Phi_{A}^{-1}(1) \\
& =\frac{1}{\Gamma_{A} \Gamma_{D}} \int_{\Phi_{D}^{-1}(0)} \int_{\Phi_{A}^{-1}\left[\Psi\left(\gamma_{D}\right)\right]} \exp \left(-\frac{\gamma_{A}}{\Gamma_{A}}-\frac{\gamma_{D}}{\Gamma_{D}}\right) \\
& {\left[1-\exp \left(-\frac{\Phi_{B}^{-1}\left[\Psi\left(\gamma_{A}, \gamma_{D}\right)\right]}{\Gamma_{B}}\right)\right] d_{\gamma_{A}} d_{\gamma_{D}}}
\end{aligned}
$$

where $\Psi\left(\gamma_{D}\right)=2 H_{b}\left(p_{A B}\right)-\Phi_{D}\left(\gamma_{D}\right)-H_{b}\left[p_{A B} * H_{b}^{-1}\left[H_{b}\left(p_{A B}\right)-\right.\right.$ $\left.\left.\Phi_{D}\left(\gamma_{D}\right)\right]\right]$ and $\Psi\left(\gamma_{A}, \gamma_{D}\right)=1+\Psi\left(\gamma_{D}\right)-\Phi_{A}\left(\gamma_{A}\right)$.

\section{APPENDIX D}

Supporting EQUATIONS For OUTAGE PRobability DERIVATION OF $M 3$

$$
\begin{aligned}
\mathbb{P}^{\prime}\left\{\mathcal{B}_{c} \mid p_{e}=0\right\} & \int_{D}^{\Phi_{D}^{-1}(\infty)} \int_{\Phi_{D}^{-1}\left[H_{b}\left(p_{A B C}\right)\right]}^{\Phi_{C}^{-1}(1) \Phi_{C}^{-1}(0)} \int_{B}^{-1} \int_{(0)}^{-1}(1) \Phi_{A}^{-1}(0) \\
= & \exp \left(-\frac{\Phi_{D}^{-1}(1)}{\Gamma_{D}}\right)\left[1-\exp \left(-\frac{\Phi_{C}^{-1}(1)}{\Gamma_{C}}\right)\right] \\
& {\left[1-\exp \left(-\frac{\Phi_{B}^{-1}(1)}{\Gamma_{B}}\right)\right]\left[1-\exp \left(-\frac{\Phi_{A}^{-1}(1)}{\Gamma_{A}}\right)\right] }
\end{aligned}
$$




$$
\begin{aligned}
\mathbb{P}^{\prime}\left\{\mathcal{B}_{t_{1}} \mid p_{e}=0\right\} & \int_{\Phi_{D}^{-1}\left[H_{b}\left(p_{A B C}\right)\right]}^{\Phi_{D}^{-1}(\infty)} \int_{\Phi_{C}^{-1}\left[H_{b}\left(p_{A B}\right)+H_{b}\left(p_{B C}\right)-2\right]}^{\Phi_{C}^{-1}(1)} \int_{\Phi_{B}^{-1}\left[\Psi\left(\gamma_{C}\right)\right]}^{\Phi_{B}^{-1}(1)} \\
& \int_{\Phi_{A}^{-1}\left[\Psi\left(\gamma_{B}, \gamma_{C}\right)\right]}^{\Phi_{A}^{-1}(1)} p\left(\gamma_{A}\right) p\left(\gamma_{B}\right) p\left(\gamma_{C}\right) p\left(\gamma_{D}\right) d_{\gamma_{A}} d_{\gamma_{B}} d_{\gamma_{C}} d_{\gamma_{D}} \\
= & \left(\Gamma_{B} \Gamma_{C}\right)^{-1} \int_{C}^{\Phi_{C}^{-1}(1)} \int_{C}^{\Phi_{B}^{-1}(1)} \exp \left(-\frac{\Phi_{D}^{-1}(1)}{\Gamma_{D}}-\frac{\gamma_{C}}{\Gamma_{C}}-\frac{\gamma_{B}}{\Gamma_{B}}\right. \\
& {\left[\exp \left(-\frac{\Phi_{A}^{-1}\left[\Psi\left(\gamma_{B}, \gamma_{C}\right)\right]}{\Gamma_{A}}\right)-\exp \left(-\frac{\Phi_{A}^{-1}(1)}{\Gamma_{A}}\right)\right] d_{\gamma_{B}} d_{\gamma_{C}}, }
\end{aligned}
$$

with $\Psi\left(\gamma_{C}\right)=H_{b}\left(p_{A B}\right)+H_{b}\left(p_{B C}\right)-1-\Phi_{C}\left(\gamma_{C}\right), \Psi\left(\gamma_{B}, \gamma_{C}\right)=$ $H_{b}\left(p_{A B}\right)+H_{b}\left(p_{B C}\right)-\Phi_{B}\left(\gamma_{B}\right)-\Phi_{C}\left(\gamma_{C}\right)$, and

$$
\mathbb{P}^{\prime}\left\{\mathcal{B}_{c} \mid 0<p_{e} \leq 0.5\right\}
$$

$$
\begin{aligned}
& \Phi_{D}^{-1}\left[H_{b}\left(p_{A B C}\right)\right] \Phi_{C}^{-1}(1) \Phi_{B}^{-1}(1) \Phi_{A}^{-1}(1) \\
& =\int_{\Phi_{D}^{-1}(0)} \int_{\Phi_{C}^{-1}(0)} \int_{\Phi_{B}^{-1}(0)} \int_{\Phi_{A}^{-1}(0)} p\left(\gamma_{A}\right) \cdots p\left(\gamma_{D}\right) d_{\gamma_{A}} \cdots d_{\gamma_{D}} \\
& =\left[1-\exp \left(-\frac{\Phi_{D}^{-1}(1)}{\Gamma_{D}}\right)\right]\left[1-\exp \left(-\frac{\Phi_{C}^{-1}(1)}{\Gamma_{C}}\right)\right] \\
& {\left[1-\exp \left(-\frac{\Phi_{B}^{-1}(1)}{\Gamma_{B}}\right)\right]\left[1-\exp \left(-\frac{\Phi_{A}^{-1}(1)}{\Gamma_{A}}\right)\right] \text {, }} \\
& \Phi_{D}^{-1}\left[H_{b}\left(p_{A B C}\right)\right] \Phi_{C}^{-1}(1) \quad \Phi_{B}^{-1}(1) \\
& \mathbb{P}^{\prime}\left\{\mathcal{B}_{t_{1}} \mid 0<p_{e} \leq 0.5\right\}=\int_{\Phi_{D}^{-1}(0)} \int_{\Phi_{C}^{-1}\left[\Psi\left(\gamma_{D}\right)\right]} \int_{\Phi_{B}^{-1}\left[\Psi\left(\gamma_{C}, \gamma_{D}\right)\right]} \\
& \Phi_{A}^{-1}(1) \\
& \int p\left(\gamma_{A}\right) p\left(\gamma_{B}\right) p\left(\gamma_{C}\right) p\left(\gamma_{D}\right) d_{\gamma_{A}} d_{\gamma_{B}} d_{\gamma_{C}} d_{\gamma_{D}}, \\
& \Phi_{A}^{-1}\left[\Psi\left(\gamma_{B}, \gamma_{C}, \gamma_{D}\right)\right] \\
& \Phi_{D}^{-1}(1) \quad \Phi_{C}^{-1}(1) \quad \Phi_{B}^{-1}(1) \\
& =\frac{1}{\Gamma_{B} \Gamma_{C} \Gamma_{D}} \int_{\Phi_{D}^{-1}(0)} \int_{\Phi_{C}^{-1}\left[\Psi\left(\gamma_{D}\right)\right]} \int_{\Phi_{B}^{-1}\left[\Psi\left(\gamma_{C}, \gamma_{D}\right)\right]} \exp \left(-\frac{\gamma_{B}}{\Gamma_{B}}-\frac{\gamma_{C}}{\Gamma_{C}}-\frac{\gamma_{D}}{\Gamma_{D}}\right) \\
& {\left[\exp \left(-\frac{\Phi_{A}^{-1}\left[\Psi\left(\gamma_{B}, \gamma_{C}, \gamma_{D}\right)\right]}{\Gamma_{A}}\right)-\exp \left(-\frac{\Phi_{A}^{-1}(1)}{\Gamma_{A}}\right)\right] d \gamma_{\gamma_{B}} d_{\gamma_{C}} d_{\gamma_{D}} \text {, }}
\end{aligned}
$$

with $\Psi\left(\gamma_{D}\right)=H_{b}\left(p_{A B}\right)+H_{b}\left(p_{B C}\right)-\Phi_{D}\left(\gamma_{D}\right)-1, \quad \Psi\left(\gamma_{C}, \gamma_{D}\right)=$ $H_{b}\left(p_{A B}\right)+H_{b}\left(p_{B C}\right)-\Phi_{D}\left(\gamma_{D}\right)-\Phi_{C}\left(\gamma_{C}\right)$, and $\Psi\left(\gamma_{B}, \gamma_{C}, \gamma_{D}\right)=$ $1+H_{b}\left(p_{A B}\right)+H_{b}\left(p_{B C}\right)-\Phi_{D}\left(\gamma_{D}\right)-\Phi_{B}\left(\gamma_{B}\right)-\Phi_{C}\left(\gamma_{C}\right)$.
The probability expressions in (29) can be expanded as:

$$
\begin{gathered}
\mathbb{P}^{\prime}\left\{\mathcal{B}_{t_{2}}^{C} \mid p_{e}=0\right\}=\int_{\substack{\Phi_{D}^{-1}\left[H_{b}\left(p_{A B C}\right)\right] \\
\Phi_{A}^{-1}\left[\Psi\left(\gamma_{B}, \gamma_{C}\right)\right]}}^{\Phi_{D}^{-1}(\infty)} \int_{\Phi_{C}^{-1}(0)}^{\Phi_{C}^{-1}\left[H_{b}\left(p_{A B}\right)+H_{b}\left(p_{B C}\right)\right]} \int_{\Phi_{B}^{-1}(0)}^{\Phi_{B}^{-1}\left[\Psi\left(\gamma_{C}\right)\right]} \\
\int_{\Phi_{A}^{-1}(0)} p\left(\gamma_{A}\right) p\left(\gamma_{B}\right) p\left(\gamma_{C}\right) p\left(\gamma_{D}\right) d_{\gamma_{A}} d_{\gamma_{B}} d_{\gamma_{C}} d_{\gamma_{D}}, \\
=\left(\Gamma_{B} \Gamma_{C}\right)^{-1} \int_{\Phi_{C}^{-1}(0)}^{\Phi_{C}^{-1}\left[H_{b}\left(p_{A B}\right)+H_{b}\left(p_{B C}\right)\right] \Phi_{B}^{-1}\left[\Psi\left(\gamma_{C}\right)\right]} \int_{\Phi_{B}^{-1}(0)} \exp \left(-\frac{\Phi_{D}^{-1}(1)}{\Gamma_{D}}-\frac{\gamma_{C}}{\Gamma_{C}}-\frac{\gamma_{B}}{\Gamma_{B}}\right) \\
{\left[1-\exp \left(-\frac{\Phi_{A}^{-1}\left[\Psi\left(\gamma_{B}, \gamma_{C}\right)\right]}{\Gamma_{A}}\right)\right] d_{\gamma_{B}} d_{\gamma_{C}},}
\end{gathered}
$$

with $\Psi\left(\gamma_{C}\right)=H_{b}\left(p_{A B}\right)+H_{b}\left(p_{B C}\right)-\Phi_{C}\left(\gamma_{C}\right), \Psi\left(\gamma_{B}, \gamma_{C}\right)=$ $H_{b}\left(p_{A B}\right)+H_{b}\left(p_{B C}\right)-\Phi_{B}\left(\gamma_{B}\right)-\Phi_{C}\left(\gamma_{C}\right)$,

$$
\begin{gathered}
\mathbb{P}^{\prime}\left\{\mathcal{B}_{t_{2}}^{C} \mid 0<p_{e} \leq 0.5\right\}=\int_{\Phi_{D}^{-1}(0)}^{\Phi_{D}^{-1}\left[H_{b}\left(p_{A B C}\right)\right]} \int_{\Phi_{C}^{-1}(0)}^{\Phi_{C}^{-1}\left[\Psi\left(\gamma_{D}\right)\right]} \int_{\Phi_{B}^{-1}(0)}^{\Phi_{B}^{-1}\left[\Psi\left(\gamma_{C}, \gamma_{D}\right)\right]} \\
\int_{\Phi_{A}^{-1}(0)}^{\Phi_{A}^{-1}\left[\Psi\left(\gamma_{B}, \gamma_{C}, \gamma_{D}\right)\right]} p\left(\gamma_{A}\right) p\left(\gamma_{B}\right) p\left(\gamma_{C}\right) p\left(\gamma_{D}\right) d_{\gamma_{A}} d_{\gamma_{B}} d_{\gamma_{C}} d_{\gamma_{D}}, \\
=\frac{1}{\Gamma_{B} \Gamma_{C} \Gamma_{D}} \int_{\Phi_{D}^{-1}(0)}^{\Phi_{D}^{-1}(1) \int_{C}^{\Phi_{C}^{-1}\left[\Psi\left(\gamma_{D}\right)\right]} \int_{B}^{-1}\left[\Psi\left(\gamma_{C}, \gamma_{D}\right)\right]} \int_{\Phi_{B}^{-1}(0)}^{\exp \left(-\frac{\gamma_{B}}{\Gamma_{B}}-\frac{\gamma_{C}}{\Gamma_{C}}-\frac{\gamma_{D}}{\Gamma_{D}}\right)} \\
{\left[1-\exp \left(-\frac{\Phi_{A}^{-1}\left[\Psi\left(\gamma_{B}, \gamma_{C}, \gamma_{D}\right)\right]}{\Gamma_{A}}\right)\right] d_{\gamma_{B}} d_{\gamma_{C}} d_{\gamma_{D}},}
\end{gathered}
$$

with $\Psi\left(\gamma_{D}\right)=1+H_{b}\left(p_{A B}\right)+H_{b}\left(p_{B C}\right)-\Phi_{D}\left(\gamma_{D}\right)$, $\Psi\left(\gamma_{C}, \gamma_{D}\right)=1+H_{b}\left(p_{A B}\right)+H_{b}\left(p_{B C}\right)-\Phi_{D}\left(\gamma_{D}\right)-\Phi_{C}\left(\gamma_{C}\right)$, and $\Psi\left(\gamma_{B}, \gamma_{C}, \gamma_{D}\right)=1+H_{b}\left(p_{A B}\right)+H_{b}\left(p_{B C}\right)-\Phi_{D}\left(\gamma_{D}\right)-\Phi_{B}\left(\gamma_{B}\right)-$ $\Phi_{C}\left(\gamma_{C}\right)$.

\section{REFERENCES}

[1] M. Agiwal, A. Roy, and N. Saxena, "Next generation 5G wireless networks: A comprehensive survey," IEEE Communications Surveys Tutorials, vol. PP, no. 99, pp. 1-1, 2016.

[2] L. Toni, T. Maugey, and P. Frossard, "Correlation-aware packet scheduling in multi-camera networks," Multimedia, IEEE Transactions on, vol. 16, no. 2, pp. 496-509, Feb 2014.

[3] G. Cheung, A. Ortega, and N.-M. Cheung, "Generation of redundant frame structure for interactive multiview streaming," in Packet Video Workshop, 2009. PV 2009. 17th International, May 2009, pp. 1-10.

[4] ITU, "High efficiency video coding," ITU-T H.265 1.0 (2013-04).

[5] M. Wien, High Efficiency Video Coding - Coding Tools and Specifications. Springer, 2014.

[6] T. K. Tan, M. Mrak, V. Baroncini, and N. Ramzan, "HEVC verification test results," Available at https://phenix.int-evry.fr/jct/doc_end_user/ current_document.php?id=9024, 17thMeeting:Joint Collaborative Team on Video Coding (JCT-VC) of ITU-T SG 16 WP 3 and ISO/IEC JTC 1/SC 29/WG 11, 2014. 
[7] S. ten Brink, G. Kramer, and A. Ashikhmin, "Design of low-density parity-check codes for modulation and detection," IEEE Transactions on Communications, vol. 52, no. 4, pp. 670-678, April 2004.

[8] K. Rao, Z. S. Bojkovic, and D. A. Milovanovic, Wireless Multimedia Communications: Convergence, DSP, QoS, and Security, 1st ed. Boca Raton, FL, USA: CRC Press, Inc., 2008.

[9] H. O. Burton and D. D. Sullivan, "Errors and error control," Proceedings of the IEEE, vol. 60, no. 11, pp. 1293-1301, Nov 1972.

[10] S. Kallel, "Analysis of a type II hybrid ARQ scheme with code combining," IEEE Transactions on Communications, vol. 38, no. 8, pp. 1133-1137, Aug 1990.

[11] Q. Chen and P. Fan, "On the performance of type-III hybrid ARQ with RCPC codes," in Personal, Indoor and Mobile Radio Communications, 2003. PIMRC 2003. 14th IEEE Proceedings on, vol. 2, Sept 2003, pp. 1297-1301 vol.2.

[12] M. W. E. Bahri, H. Boujernaa, and M. Siala, "Performance comparison of type I, II and III hybrid ARQ schemes over AWGN channels," in Industrial Technology, 2004. IEEE ICIT '04. 2004 IEEE International Conference on, vol. 3, Dec 2004, pp. 1417-1421 Vol. 3.

[13] L. Szczecinski, S. R. Khosravirad, P. Duhamel, and M. Rahman, "Rate allocation and adaptation for incremental redundancy truncated HARQ," IEEE Transactions on Communications, vol. 61, no. 6, pp. 2580-2590, June 2013.

[14] R. Palanki and J. S. Yedidia, "Rateless codes on noisy channels," Available at www.merl.com/papers/TR2003-124/.

[15] K. Wu, Z. Zhang, Y. Huo, and S. Yang, "Accumulate rateless codes for AWGN channel," in Communications and Networking in China, 2008. ChinaCom 2008. Third International Conference on, Aug 2008, pp. 100 104.

[16] S. Kokalj-Filipovic, P. Spasojevic, E. Soljanin, and R. Yates, "Arq with doped fountain decoding," in 2008 IEEE 10th International Symposium on Spread Spectrum Techniques and Applications, Aug 2008, pp. 780784.

[17] D. Nguyen, T. Tran, T. Nguyen, and B. Bose, "Wireless broadcast using network coding," IEEE Transactions on Vehicular Technology, vol. 58, no. 2, pp. 914-925, Feb 2009.

[18] P. Larsson, B. Smida, T. Koike-Akino, and V. Tarokh, "Analysis of network coded HARQ for multiple unicast flows," IEEE Transactions on Communications, vol. 61, no. 2, pp. 722-732, February 2013.

[19] Z. Li, Q. Luo, and W. Featherstone, "N-in-1 retransmission with network coding," Wireless Communications, IEEE Transactions on, vol. 9, no. 9, pp. 2689-2694, September 2010.

[20] A. Wyner, "On source coding with side information at the decoder," IEEE Transactions on Information Theory, vol. 21, no. 3, pp. 294-300, May 1975.

[21] R. Ahlswede and J. Korner, "Source coding with side information and a converse for degraded broadcast channels," IEEE Transactions on Information Theory, vol. 21, no. 6, pp. 629-637, Nov 1975.

[22] A. Gamal and Y. Kim, Network Information Theory. Cambridge University Press, 2011.

[23] X. Zhou, P. S. Lu, K. Anwar, and T. Matsumoto, "Correlated sources transmission in orthogonal multiple access relay channel: Theoretical analysis and performance evaluation," IEEE Transactions on Wireless Communications, vol. 13, no. 3, pp. 1424-1435, March 2014.

[24] J. Garcia-Frias and Y. Zhao, "Near-Shannon/Slepian-Wolf performance for unknown correlated sources over AWGN channels," IEEE Transactions on Communications, vol. 53, no. 4, pp. 555-559, April 2005. 